\title{
Effective Radii of Young, Massive Star Clusters in Two LEGUS Galaxies*
}

\author{
J. E. Ryon ${ }^{1,2}$, J. S. Gallagher ${ }^{1}$, L. J. Smith ${ }^{3}$, A. Adamo ${ }^{4}$, D. Calzetti ${ }^{5}$, S. N. Bright ${ }^{2}$, M. Cignoni ${ }^{2,6}$, D. O. Cook ${ }^{7,8}$, D. A. Dale ${ }^{7}$, \\ B. E. Elmegreen ${ }^{9}$, M. Fumagalli ${ }^{10}$, D. A. Gouliermis ${ }^{11,12}$, K. Grasha ${ }^{5}$, E. K. Grebel ${ }^{13}$, H. Kim ${ }^{14}$, M. Messa ${ }^{4}$, D. Thilker ${ }^{15}$, and \\ L. Ubeda ${ }^{2}$ \\ ${ }^{1}$ University of Wisconsin-Madison, 475 N. Charter St., Madison, WI 53706, USA \\ ${ }^{2}$ Space Telescope Science Institute, 3700 San Martin Dr., Baltimore, MD 21218, USA \\ ${ }^{3}$ European Space Agency/Space Telescope Science Institute, 3700 San Martin Dr., Baltimore, MD 21218, USA \\ ${ }_{5}^{4}$ Dept. of Astronomy, The Oskar Klein Centre, Stockholm University, Stockholm, Sweden \\ ${ }^{5}$ Dept. of Astronomy, University of Massachusetts-Amherst, Amherst, MA 01003, USA \\ ${ }^{6}$ Department of Physics, University of Pisa, Largo Pontecorvo, 3 Pisa, I-56127, Italy \\ ${ }^{7}$ Dept. of Physics and Astronomy, University of Wyoming, Laramie, WY, USA \\ ${ }^{8}$ California Institute of Technology, 1200 East California Blvd, Pasadena, CA 91125, USA \\ ${ }^{9}$ IBM Research Division, T.J. Watson Research Center, Yorktown Hts., NY, USA \\ ${ }^{10}$ Institute for Computational Cosmology and Centre for Extragalactic Astronomy, Durham University, Durham, UK \\ ${ }^{11}$ Zentrum für Astronomie der Universität Heidelberg, Institut für Theoretische Astrophysik, Albert-Ueberle-Str. 2, D-69120 Heidelberg, Germany \\ ${ }^{12}$ Max Planck Institute for Astronomy, Königstuhl 17, D-69117 Heidelberg, Germany \\ ${ }^{13}$ Astronomisches Rechen-Institut, Zentrum für Astronomie der Universität Heidelberg, Mönchhofstr. 12-14, D-69120 Heidelberg, Germany \\ ${ }^{14}$ Gemini Observatory, Casilla 603, La Serena, Chile \\ 15 Dept. of Physics and Astronomy, The Johns Hopkins University, Baltimore, MD, USA \\ Received 2017 February 25; revised 2017 April 28; accepted 2017 April 30; published 2017 May 30
}

\begin{abstract}
We present a study of the effective (half-light) radii and other structural properties of a systematically selected sample of young, massive star clusters $\left(\geqslant 5 \times 10^{3} M_{\odot}\right.$ and $\left.\leqslant 200 \mathrm{Myr}\right)$ in two nearby spiral galaxies, NGC 628 and NGC 1313. We use Hubble Space Telescope (HST) WFC3/UVIS and archival ACS/WFC data obtained by the Legacy Extragalactic UV Survey (LEGUS), an HST Treasury Program. We measure effective radii with GALFIT, a two-dimensional image-fitting package, and with a new technique to estimate effective radii from the concentration index of observed clusters. The distribution of effective radii from both techniques spans $\sim 0.5-10$ pc and peaks at 2-3 pc for both galaxies. We find slight positive correlations between effective radius and cluster age in both galaxies, but no significant relationship between effective radius and galactocentric distance. Clusters in NGC 1313 display a mild increase in effective radius with cluster mass, but the trend disappears when the sample is divided into age bins. We show that the vast majority of the clusters in both galaxies are much older than their dynamical times, suggesting they are gravitationally bound objects. We find that about half of the clusters in NGC 628 are underfilling their Roche lobes, based on their Jacobi radii. Our results suggest that the young, massive clusters in NGC 628 and NGC 1313 are expanding, due to stellar mass loss or two-body relaxation, and are not significantly influenced by the tidal fields of their host galaxies.
\end{abstract}

Key words: galaxies: general - galaxies: individual (NGC 628, NGC 1313) - galaxies: star clusters: general

Supporting material: machine-readable tables

\section{Introduction}

The discovery of young, massive clusters (YMCs) residing in nearby galaxies has spurred major interest in recent decades in determining their properties and evolution. The sizes of YMCs appear to be nearly constant across a wide range of age, mass, and environment (e.g., Portegies Zwart et al. 2010). The radius containing one-half of the total cluster light, the effective radius or $r_{\text {eff }}$, is the most straightforward size scale to measure observationally and is typically found to be 2-3 pc for YMCs (e.g., Elson et al. 1987; Whitmore et al. 1999; Larsen 2004; Barmby et al. 2006; Scheepmaker et al. 2007; Portegies Zwart et al. 2010; Bastian et al. 2012). Interestingly, globular clusters (GCs) also have characteristic effective radii of 2-3 pc (e.g., Jordán et al. 2005; Harris 2009; Masters et al. 2010; Puzia et al. 2014). If today's YMCs are modern-day progenitors of

\footnotetext{
* Based on observations obtained with the NASA/ESA Hubble Space Telescope, at the Space Telescope Science Institute, which is operated by the Association of Universities for Research in Astronomy, Inc., under NASA contract NAS 5-26555. These observations are associated with program \#13364.
}

ancient GCs, then studying YMC sizes and evolution locally may shed light on the origins of GCs.

The size of a star cluster is tied to the internal and external mechanisms that influence the dynamical state of the stars in the cluster. A better understanding of the sizes of YMCs can therefore constrain their formation and early evolution. For instance, the relationship between cluster age and radius may show whether YMCs of a certain age and mass range are expanding or contracting, which would indicate whether they are behaving as isolated or tidally limited systems (Heggie \& Hut 2003; Trenti et al. 2010; Alexander \& Gieles 2013). Similarly, the dependence of cluster radius on distance from the galaxy center may provide clues to the influence of the galaxy's tidal field on YMC evolution (Gieles et al. 2011; Madrid et al. 2012; Alexander \& Gieles 2013; Sun et al. 2016). The relationship between cluster mass and radius may illuminate the effect of perturbations by giant molecular clouds (GMCs). For a weak mass-radius relationship, which has been found by several studies (e.g., Zepf et al. 1999; Larsen 2004; Scheepmaker et al. 2007; Barmby et al. 2009), less massive objects are more likely to be disrupted by GMC interactions because they are 
of lower density (Gieles et al. 2006). In addition, Gieles \& Portegies Zwart (2011) show it is possible to determine whether an object is likely to be gravitationally bound (star clusters) or unbound (associations) by comparing the crossing time, calculated from mass and radius, to the object's age.

One of the central goals of the Legacy Extragalactic UV Survey (LEGUS; Calzetti et al. 2015) is to better understand the role of star clusters in the star formation process. Part of this goal is to determine the shape of the cluster radius distribution and whether it depends on galaxy environment. In this paper, we directly address this goal by studying the sizes of homogeneously selected YMCs in two LEGUS galaxies, NGC 628 and NGC 1313. These two galaxies were chosen for this study because of their relatively numerous cluster populations and differing morphological types. They provide an interesting contrast to probe effects of the environment on cluster structure. In addition, NGC 1313 is half the distance of NGC 628, and we can therefore test how spatial resolution affects the measured cluster properties.

NGC 628 (M74) is a face-on ( $\left.i=25^{\circ} .2\right)$ grand-design spiral galaxy (SAc) located at a distance of $9.9 \pm 1.3 \mathrm{Mpc}$ (Olivares et al. 2010). It has a stellar mass of $1.1 \times 10^{10} M_{\odot}$ and an extinction-corrected UV star formation rate (SFR) of 3.67 $M_{\odot} \mathrm{yr}^{-1}$ (Calzetti et al. 2015). Thilker et al. (2007) noted the presence of an extended UV disk featuring a spiral structure that is a continuation of the inner, optically bright pattern. Though it is the largest member of a galaxy group, the regular appearance of its disk suggests no recent interactions. Adamo et al. (2017) provide an overview of the cluster analysis techniques employed by LEGUS and present results on the luminosity function, mass function, and age distribution of the YMC population of NGC 628 as a test case. Larsen (2004) measured effective radii for 30 clusters in NGC 628 and found an average radius of $3.65 \pm 0.55 \mathrm{pc}$.

NGC 1313 is a somewhat-inclined ( $i=40.7)$ SBd galaxy located at a distance of $4.39 \pm 0.04 \mathrm{Mpc}$ (Jacobs et al. 2009). Its stellar mass is $2.6 \times 10^{9} M_{\odot}$, and the extinction-corrected UV SFR is $1.15 M_{\odot} \mathrm{yr}^{-1}$ (Calzetti et al. 2015). The resemblance between NGC 1313 and the Large Magellanic Cloud has been noted previously (de Vaucouleurs 1963), given its bar and rather irregular appearance. A number of studies suggest that NGC 1313 may be interacting with a satellite galaxy, which has produced a loop of $\mathrm{HI}$ gas around the galaxy (Peters et al. 1994) and led to an increase in SFR in the southwestern part of the galaxy over the past $100 \mathrm{Myr}$ (Silva-Villa \& Larsen 2012). Previous studies have noted strong evidence for disruption of young clusters (Pellerin et al. 2007), a high cluster formation rate (Silva-Villa \& Larsen 2011), and cluster radii between $\sim 2$ and 5 pc, on average (Larsen 2004; Mora et al. 2009).

This work builds upon the techniques and results from Ryon et al. (2015), in which we measured the effective radii and light profile slopes of $\sim 200$ YMCs in the nearby spiral galaxy M83 using GALFIT, a two-dimensional image-fitting package. In this paper, we select clusters from two adjacent $H S T$ fields obtained by LEGUS for each of NGC 628 and NGC 1313, resulting in samples of 320 and 195 YMCs, respectively. We fit these clusters with GALFIT to determine their effective radii and light profile slopes. Since GALFIT does not properly fit some types of clusters, we also calculate an estimate of the effective radius from the concentration index (CI) of each cluster using a relation determined from artificial clusters. We compare the effective radii and light profile shapes of the clusters to their ages, masses, and galactocentric distances to probe the mechanisms that drive their structural evolution and further investigate their dynamical states.

This paper is organized as follows. In Section 2, we describe the observations and star cluster catalog. We describe our methods for measuring effective radii and completeness tests in Section 3. In Section 4, we present the results of our measurements and explore relationships between size and other cluster properties. We briefly discuss the implications of this work and summarize our findings in Section 5.

\section{Observations and Cluster Catalogs}

LEGUS is a Hubble Space Telescope (HST) Cycle 21 Treasury program, which obtained imaging of 50 nearby galaxies (within $\sim 13 \mathrm{Mpc}$ ) in five filters with WFC3/UVIS and ACS/WFC. New imaging for selected pointings on each galaxy were obtained with WFC3/UVIS to complement archival ACS/WFC imaging and complete the multiband coverage from the near-UV to the I band. All images are drizzled to the UVIS native pixel scale of 0!.03962/pixel. See Calzetti et al. (2015) for a complete description of the data reduction of the LEGUS imaging data sets. In this study, we measure star cluster sizes from the F555W images. ${ }^{16}$

The production of catalogs of candidate star clusters by LEGUS is described in detail in Adamo et al. (2017). Here, we briefly describe the catalogs from which the cluster samples for this study were selected.

Separate catalogs are produced for each pointing on the two galaxies: NGC 628c (central pointing), NGC 628e (east pointing), NGC 1313e (east pointing), and NGC 1313w (west pointing). First, SourceExtractor (Bertin \& Arnouts 1996) identifies sources in the white-light image of each pointing (see Calzetti et al. 2015 for a description of the white-light images for LEGUS). Next, growth curves and CI values are determined for user-identified isolated stars and star cluster candidates, which allow selection of the appropriate photometric aperture size and CI value for separating stars and cluster candidates. The CI is the magnitude difference between aperture radii of 1 and 3 pixels in the $\mathrm{F} 555 \mathrm{~W}$-band image and is therefore larger for more extended objects (e.g., Holtzman et al. 1996; Whitmore et al. 2010). Aperture photometry is performed in all five filters using the science aperture radius determined from the isolated clusters and a background annulus located at seven pixels with a width of one pixel.

Average aperture corrections are calculated from the isolated clusters by measuring the average magnitude difference from the science aperture to a radius of 20 pixels in each band. We apply the aperture corrections and Galactic foreground extinction corrections to the science photometry. Finally, for each pointing, a catalog for visual inspection is produced by performing a series of cuts: each source must have a CI value larger than the CI limit determined from isolated stars and clusters, be detected in at least four bands with a photometric error below $0.3 \mathrm{mag}$, and have an absolute F555W-band magnitude brighter than -6 mag. Relevant parameters used by the LEGUS team to produce the candidate cluster catalogs for each pointing are provided in Table 1 .

At least three members of the LEGUS team visually inspected each cluster candidate that satisfies the above criteria.

\footnotetext{
${ }^{16}$ The final reduced images are available at doi:10.17909/T9J01Z.
} 
Table 1

LEGUS Cluster Catalog Parameters

\begin{tabular}{llccc}
\hline \hline & $\begin{array}{c}\text { Camera } \\
\text { F555W }\end{array}$ & $\begin{array}{c}\text { CI } \\
\text { Limit }\end{array}$ & $\begin{array}{c}\text { Aperture } \\
\text { Radius } \\
\text { (pix) }\end{array}$ & Distance \\
\hline NGC 628c & ACS & 1.4 & 4 & 9.9 \\
NGC 628e & WFC3 & 1.3 & 4 & 9.9 \\
NGC 1313e & ACS & 1.4 & 6 & 4.39 \\
NGC 1313w & ACS & 1.4 & 6 & 4.39 \\
\hline
\end{tabular}

The cluster candidate was assigned one of four classes by each LEGUS team member. The descriptions of each class are as follows:

Class 1: Compact and centrally concentrated with an FWHM more extended than that of a star. Homogeneous in color.

Class 2: Slightly elongated or asymmetric light profile shapes with an FWHM more extended than that of a star. Homogeneous in color.

Class 3: Asymmetric light profiles consisting of multiple peaks on top of diffuse underlying light.

Class 4: Spurious sources including single stars, pairs of stars (color difference), chip edge artifacts, hot pixels, and background galaxies.

The mode and mean class is determined for each source and listed in the final LEGUS catalog.

The age, mass, and extinction of each cluster candidate in the visual inspection catalogs with photometric detections in at least four filters are determined using the SED-fitting code Yggdrasil (Zackrisson et al. 2011). For this study, we use the catalogs containing fits performed with Padova-AGB isochrones, available in Starburst99 (Leitherer et al. 1999; Vázquez \& Leitherer 2005), and the Milky Way extinction law from Cardelli et al. (1989). The results of the SED fitting are listed in the final cluster candidate catalogs together with ID numbers, R.A. decl. and pixel coordinates, magnitudes, CI values, residuals from the SED fitting, reduced chi-squared of the SED fits, and visual inspection class assignments.

We impose further selection criteria to select a bona fide sample of young, massive star cluster candidates from both galaxies. First, we limit our samples to objects with a mode visual inspection class of 1 or 2 . This ensures that the light profiles are relatively well behaved and that the objects are centrally concentrated, as is expected for gravitationally bound star clusters. We also limit our sample to objects with masses $\geqslant 5000 M_{\odot}$ and ages $\leqslant 200$ Myr. The mass cut minimizes the effects of stochastic sampling of the stellar initial mass function (IMF; e.g., Fouesneau \& Lançon 2010; Popescu \& Hanson 2010), and the age cut ensures that we are not strongly affected by incomplete detection of clusters due to evolutionary fading. In Figures 1 and 2, we plot the locations of clusters that satisfy these selection criteria as open circles on grayscale F555W mosaic images of NGC 628 and NGC 1313, respectively. The blue circles are visual inspection class 1 cluster candidates, and the orange circles are class 2.

In Figure 3, we plot the ages and masses of cluster candidates in NGC 628 (top row) and NGC 1313 (bottom row) as determined from the SED fits. The left panels show the class 1 and 2 objects in each galaxy. The dashed lines represent the age and mass cuts we have applied to the samples, and the shaded regions contain all objects that we have attempted to fit

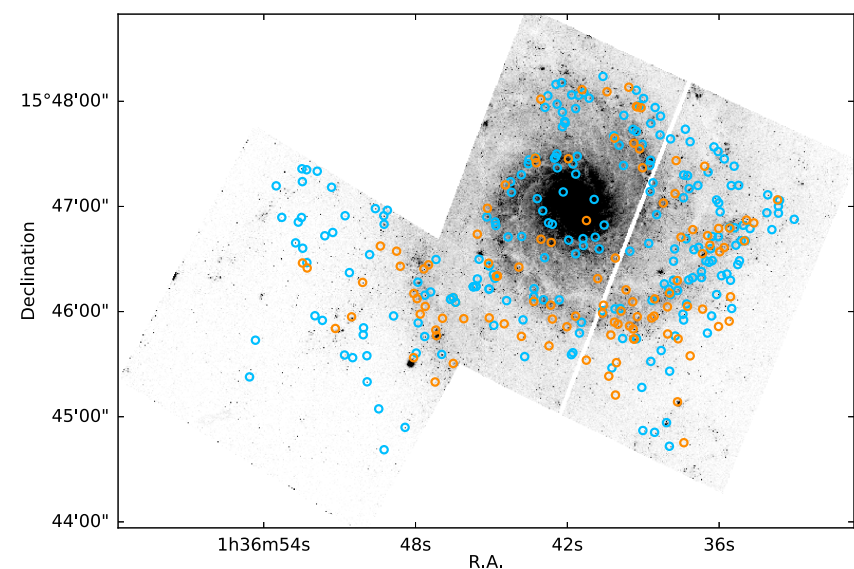

Figure 1. Grayscale F555W image of NGC 628 with the locations of cluster candidates overplotted as open circles. Class 1 objects are blue circles, and class 2 objects are orange circles.

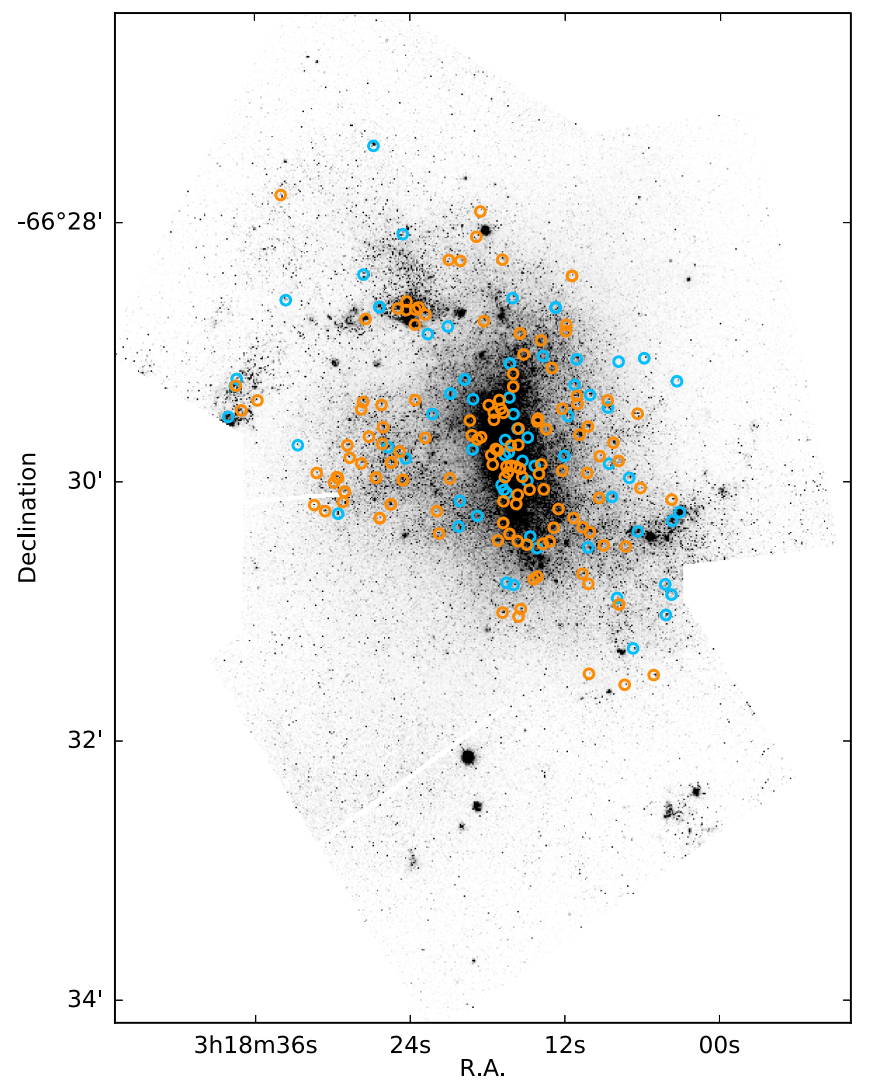

Figure 2. Same as for Figure 1, but for NGC 1313.

with GALFIT and estimated effective radii from the CI. The middle panels show the clusters successfully fit with GALFIT, and the right panels show the objects for which effective radii have been estimated from CI values. The yellow, light blue, and dark blue solid lines show the maximum, mean, and median cluster mass, respectively, in bins of width 0.1 dex in age that contain at least five objects. These data show the expected statistical correlation between cluster age and mass that results from the larger number of older star clusters that more completely sample the cluster upper mass range.

The total number of clusters remaining in the NGC 628 sample after the visual inspection class, mass, and age cuts have been applied is 320 , which consists of 257 in the central 


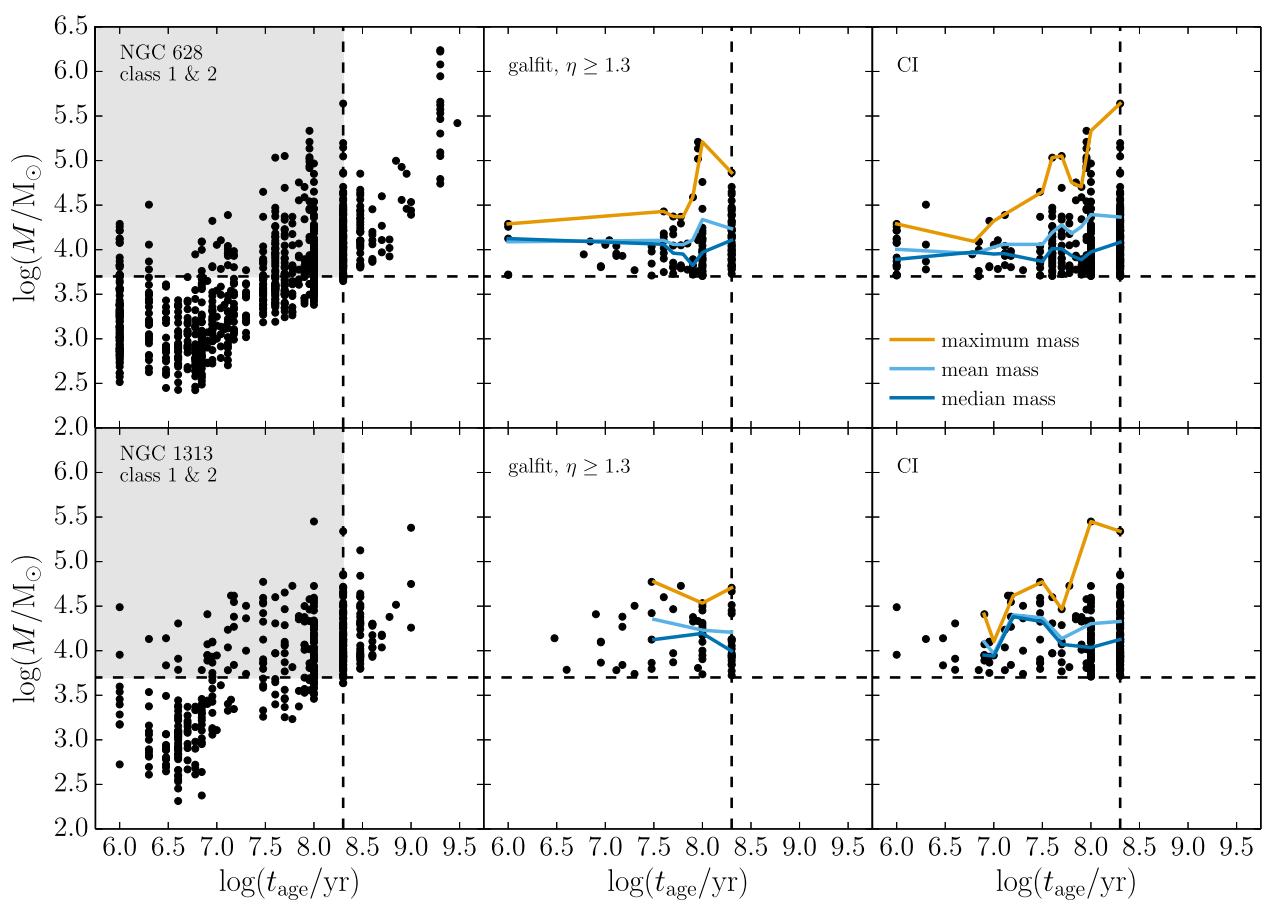

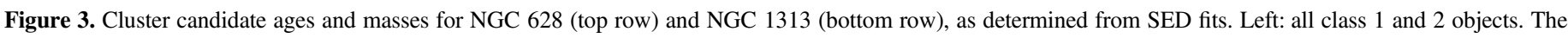

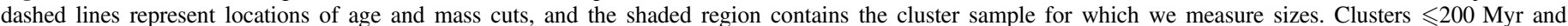

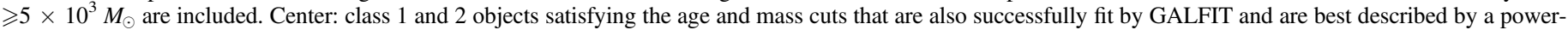

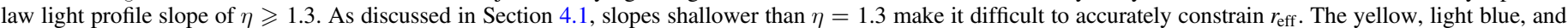

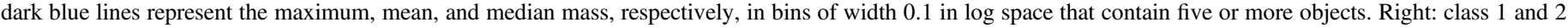
objects satisfying the age and mass cuts for which effective radii have been estimated from CI values. Lines are the same as in the center panel.

pointing and 63 in the east pointing. For NGC 1313, the total number of clusters remaining is 195 , which consists of 54 in the east pointing and 141 in the west pointing.

\section{Methods}

\subsection{Effective Radii from GALFIT}

The methods used for fitting the two-dimensional light profiles of cluster candidates with GALFIT (Peng et al. 2002, 2010) are described in detail in Paper I. We briefly summarize those methods and a few small differences here.

Other studies have used ISHAPE (Larsen 1999) to measure the structural properties of star clusters in nearby galaxies. A comparison of the results from ISHAPE and GALFIT fits of a subsample of well-behaved clusters included in Paper I found differences in measured effective radii on the order of about $6 \%$. It is therefore unlikely that the choice of fitting software would strongly affect the results we present here. We chose to use GALFIT because its reporting of fitting results and errors is somewhat clearer than ISHAPE.

To prepare the HST F555W images for use with GALFIT, we multiplied the drizzled images by the exposure time to convert from units of $\mathrm{e}^{-} / \mathrm{s}$ to $\mathrm{e}^{-}$. We also updated the image headers to set the GAIN keyword equal to $1.0 \mathrm{e}^{-} / \mathrm{ADU}$ and to include the read noise for the appropriate camera, $3.11 \mathrm{e}^{-}$for WFC3/UVIS and $4.2 \mathrm{e}^{-}$for ACS/WFC, in the header keyword RDNOISE.

In order to extract the structural components of each cluster from the LEGUS images, GALFIT convolves a model image with a point-spread function (PSF) and compares the result to the observed data. An accurate stellar PSF is essential for reproducing the effects of the telescope optics in the model images. We create PSFs for each LEGUS pointing from several bright, isolated stars in each image by using pstselect and psf within DAOPHOT in IRAF. We spatially subsample the empirical PSFs by a factor of 10 .

We assume an EFF light profile shape (also known as a Moffat profile), because it describes well the light profiles of young star clusters in particular (Elson et al. 1987; Larsen 1999; McLaughlin \& van der Marel 2005). The EFF profile takes the form

$$
\mu(r)=\mu_{0}\left(1+r^{2} / a^{2}\right)^{-\eta}
$$

where $\mu$ is the surface brightness, $a$ is a characteristic radius, and $\eta$ is the power-law exponent of the profile wings. Note that $\eta$ is equal to $\gamma / 2$ in Equation (1) of Elson et al. (1987). The effective radius $\left(r_{\text {eff }}\right)$, or half-light radius, is defined to be the radius of the circle that contains one-half of the total surface brightness of the light profile. This is written as

$$
r_{\text {eff }}=\operatorname{FWHM} \frac{\sqrt{(1 / 2)^{\frac{1}{1-\eta}}-1}}{2 \sqrt{2^{1 / \eta}-1}},
$$

which is only valid for $\eta>1.0$. For an elliptical profile, the true effective radius can be found by multiplying Equation (2) by a factor of $0.5(1+b / a)$, where $b / a$ is the semiminor to semimajor axis ratio supplied by GALFIT (ISHAPE manual, Larsen 1999).

For each cluster, both an EFF light profile and a local background component are fit simultaneously over a $30 \times 30$ pixel region centered on the cluster. This fitting region size was selected to be consistent with that used in Paper I for M83. Different fitting region sizes were tested in Paper I and found to not significantly affect the overall results (see Table 2 in that 
Table 2

GALFIT Input Parameters

\begin{tabular}{lc}
\hline \hline Parameter & Value \\
\hline Total Magnitude & $20.0 \mathrm{mag}, 17.0 \mathrm{mag}^{\mathrm{a}}$ \\
FWHM & $2.5 \mathrm{pix}$ \\
$\eta$ & 1.5 \\
Axis Ratio & 1.0 \\
Position Angle & $25^{\circ}$ \\
Background & $300.0 \mathrm{e}^{-}$ \\
\hline
\end{tabular}

Note.

${ }^{\mathrm{a}}$ The total magnitude initial guess is $20.0 \mathrm{mag}$ for NGC 628 and 17.0 mag for NGC 1313.

work). The fitting region is equivalent to $\approx 57 \times 57 \mathrm{pc}$ at the distance of NGC 628 and $\approx 25 \times 25$ pc at the distance of NGC 1313. The amplitude of the local background is left as a free parameter. The free parameters for the EFF component are the $x$ and $y$ image coordinates of the cluster center, total magnitude, FWHM, $\eta$, axis ratio $(b / a)$, and position angle. Table 2 lists the initial guesses for each of the free parameters, excluding the $x y$ coordinates, for both galaxies. GALFIT returns the best-fit values for each free parameter and their $1 \sigma$ uncertainties. At times, GALFIT cannot converge on a bestfitting model, or one of the fitting parameters becomes unphysically small. The objects for which this occurred, of which there were 46 in the NGC 628 sample and 29 in the NGC 1313 sample, are labeled in the GALFIT output file. We exclude them from the GALFIT sample.

Finally, we inspect the residual image produced by GALFIT for each cluster candidate. Clusters for which the EFF component of the fit appears to be influenced by the presence of other objects within the fitting region (e.g., stars, clusters, areas of high background) are flagged and excluded from the following analysis. Of the clusters in the NGC 628 sample, 58 were found to be affected by other objects within the fitting region. In NGC 1313, 64 clusters were affected. For some of the objects, GALFIT also could not converge on a best-fitting solution, as described above. By combining the selection criteria listed in this section and Section 2, the total number of clusters successfully fit with GALFIT can be determined. There are 241 clusters remaining in the NGC 628 sample, including 194 in the central pointing and 47 in the east pointing, and 130 remaining in the NGC 1313 sample, including 36 in the east pointing and 94 in the west pointing.

\subsection{Effective Radii Estimated from CI}

In addition to measuring effective radii using GALFIT, we have developed a method to estimate effective radii from a cluster's CI. To do this, we create artificial clusters with the LEGUS cluster completeness tool described in Adamo et al. (2017). The first step of this tool uses routines in the baolab environment (Larsen 1999) to create artificial star clusters from an input stellar PSF. The PSF is convolved with a symmetric EFF light profile with a power-law index of $\eta=1.5$ and a predetermined effective radius to produce an artificial cluster of a given size. For each input radius, a single frame is created, containing 500 artificial clusters of that radius and a range of apparent magnitudes. The magnitude ranges, which are 18-24 mag for NGC 628 and $\sim 17-23$ mag for NGC 1313 in $\mathrm{F} 555 \mathrm{~W}$, were chosen to match the magnitude range of the

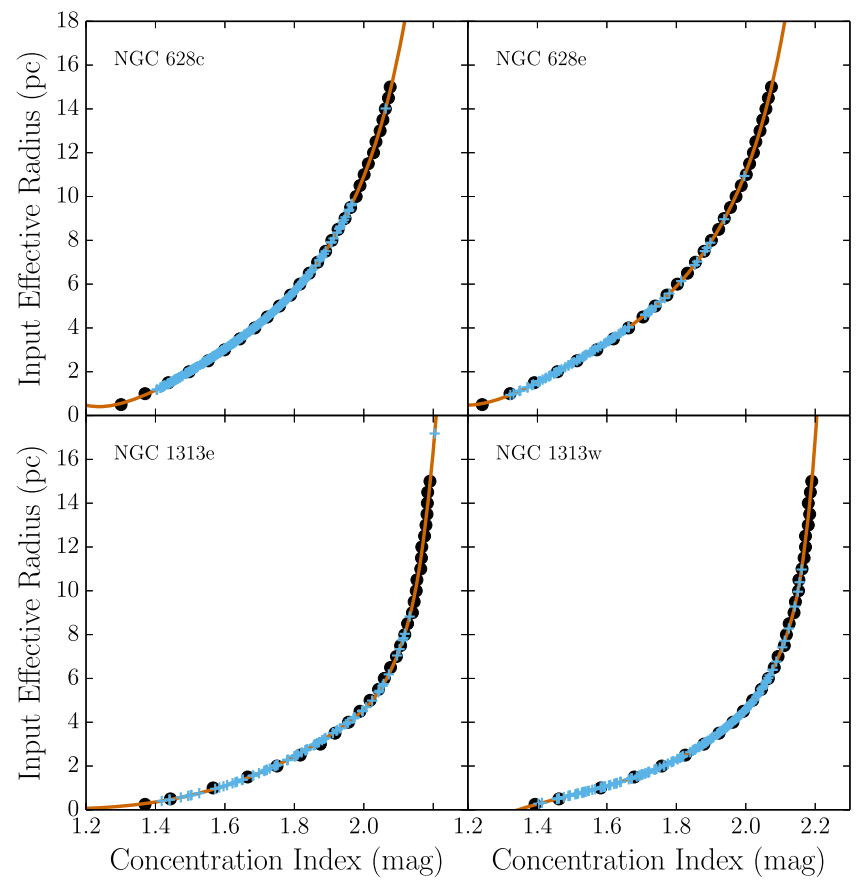

Figure 4. Input effective radius vs. measured CI for artificial clusters in the four LEGUS pointings. The black points show the median CI values of 500 artificial clusters with the same input effective radius. The orange line is the univariate spline fit to the black points. The blue crosses show the estimated effective radii of the observed clusters, calculated from the spline fit.

real clusters in each galaxy. The artificial clusters are placed randomly within the frame, which is then added to a defined region within the F555W image of a LEGUS pointing. This region is determined by finding where the WFC3/UVIS and ACS/WFC footprints overlap for each pointing. We produce images containing artificial clusters with effective radii of $0.5-15.0 \mathrm{pc}$ in steps of $0.5 \mathrm{pc}$, as well as one frame of $0.25 \mathrm{pc}$ radius clusters for each of the NGC 1313 pointings.

For each LEGUS pointing, we measure the CI of each artificial cluster in every frame using the same procedure as for the observed clusters. We calculate the median and median absolute deviation (MAD; Feigelson \& Jogesh Babu 2012) of the $\mathrm{CI}$ of the artificial clusters with each input effective radius. The range of median CI values of the artificial clusters matches that of the observed clusters well. For the NGC 628 pointings, we use the UnivariateSpline class within scipy. interpolate to fit a fourth-order univariate spline with a smoothing factor of $s=0.1$ to the median CI and input radius values. For the NGC 1313 pointings, we use the same python class to fit a third-order univariate spline with a smoothing factor of $s=1.2$ to the median $\mathrm{CI}$ and input radius values. In Figure 4, we plot the median CI and input effective radii of the artificial clusters along with the spline fit for each of the four pointings.

From this relation, we are able to estimate effective radii from the CI values of the observed clusters in each pointing. The small level of scatter in Figure 4 indicates that the CI fits are not overly sensitive to cluster ellipticity. This is consistent with other studies that show circular profiles give good results, especially for cases where the axial ratio $b / a$ is greater than 0.3 (e.g., Matthews et al. 1999; Smith \& Gallagher 2001). The difference in the overall shapes of the CI- $r_{\text {eff }}$ relationships between the two galaxies is likely a distance effect. 
Artificial clusters of the same input radii have larger CI values in NGC 1313 than in NGC 628 because the former galaxy is approximately one-half the distance of the latter. We estimate $1 \sigma$ errors on the effective radii by creating 5000 Monte Carlo realizations of the observed $\mathrm{CI}$ values assuming the $\mathrm{CI}$ photometric errors are $1 \sigma$ uncertainties and calculating the standard deviation of the resulting effective radius distribution for each observed cluster. Using this method, we are able to estimate effective radii for all of the clusters that satisfy the selection criteria listed in Section 2, that is, 320 clusters in NGC 628 and 195 in NGC 1313. As shown by the blue crosses in Figure 4, only a small fraction of observed clusters have CI values greater than 2.0. Above this value, the CI-effective radius relations become quite steep, and therefore a small uncertainty in $\mathrm{CI}$ leads to a large uncertainty in effective radius.

\subsection{Completeness Tests}

\subsubsection{Artificial Cluster Tests}

We perform tests on the artificial clusters created in Section 3.2 in order to determine if extremely compact star clusters are preferentially lost from the sample due to problems with convergence of GALFIT fits. To do this, we run GALFIT on artificial clusters in the $\mathrm{F} 555 \mathrm{~W}$ images of each pointing on NGC 628 and NGC 1313, which were created as described in Section 3.2. We consider a subset of those images containing artificial clusters with effective radii ranging between 0.5 and $15 \mathrm{pc}$ for NGC 628 and 0.25 and $15 \mathrm{pc}$ for NGC 1313. The initial guesses for the GALFIT fits are the same as for the observed star clusters (see Table 2).

Next, we perform aperture photometry on the artificial clusters in the same manner as for the observed star clusters. For bins in magnitude of width $0.5 \mathrm{mag}$, we find the number of artificial clusters that are "recovered" by GALFIT, which means that their fits successfully converged. This is done for each input effective radius. We then divide the number of recovered clusters by the total to get the percentage of objects recovered in each magnitude bin for every effective radius. Photometric blends were not removed. We calculate errors by taking the square root of the number of clusters "recovered" in each bin, dividing by the total number input into GALFIT, and converting to a percentage. The total number of clusters input into GALFIT summing over all magnitude bins is 500, and the errors become very large when small numbers of clusters are recovered.

We plot the results in Figure 5. In NGC 628c and NGC 628e (Figures 5(a) and (b), respectively), the majority of clusters in the $r_{\text {eff }}=0.5 \mathrm{pc}$ bin are not recovered, probably because these objects straddle the boundary between unresolved stars and semiresolved clusters. The recovery rate of clusters with $1.0 \mathrm{pc}$ radii varies significantly over the magnitude range, between $30 \%$ and $70 \%$ for NGC 628c, and $40 \%$ and $80 \%$ for NGC 628e. For objects in the $r_{\text {eff }}=1.5 \mathrm{pc}$ bin, the recovery percentage is $100 \%$ at the bright end of the magnitude range, then begins to decrease to 50\%-60\% for both NGC 628 pointings, though the falloff occurs more rapidly for NGC 628c. However, the majority of 1.5 pc clusters in both pointings are recovered. For clusters with input $r_{\text {eff }} \geqslant 2.0 \mathrm{pc}$, the recovery rate is essentially $100 \%$ in all magnitude bins, except for some dips at the faint end in the larger radius panels. This indicates that GALFIT recovers almost all of the clusters greater than $2.0 \mathrm{pc}$ in radius in both pointings and the majority of sources between 1.0 and $2.0 \mathrm{pc}$ in effective radius.

For each input radius, the recovery percentages are almost identical between the two NGC 1313 pointings (Figures 5(c) and (d)). The majority of the 0.25 pc clusters are not recovered, again probably because they are close to the resolution limit between stars and clusters. The brightest objects in the $0.5 \mathrm{pc}$ panels are $100 \%$ recovered and followed by a falloff to $30 \%-40 \%$ recovered at the faintest magnitudes, but the majority of objects are recovered. Above $1.0 \mathrm{pc}$ radii, essentially all objects are recovered, except for dips to $\sim 80 \%$ at the fainter magnitudes. For NGC 1313, GALFIT is able to recover the majority of sources between 0.5 and $1.0 \mathrm{pc}$ and almost all sources larger than $1.0 \mathrm{pc}$ in radius. The difference between recovery rates for artificial clusters with small radii for the two galaxies is likely due to the fact that NGC 628 is about twice as far away as NGC 1313.

\subsubsection{Bright Objects in LEGUS Stellar Catalogs}

The artificial cluster tests in Section 3.3.1 assume that the sample input into GALFIT is not already biased against very compact clusters. To test this assumption, we search the final cluster catalog of each pointing for objects that satisfy $M_{V} \leqslant-6, M \geqslant 5000 M_{\odot}$, age $\leqslant 200 \mathrm{Myr}$, and visual inspection class (mode) $\neq 1$ or 2 . With this sample, we look for objects that may have been misclassified as stars (class 4) or associations (class 3) that could actually be very compact clusters that would fall in our parameter space. We also search the LEGUS version 1 stellar catalog (E. Sabbi et al. 2017, in preparation) for objects that satisfy $M_{V} \leqslant-8$. Very few stars are brighter than this absolute magnitude limit (Massey et al. 2006), so any objects in the catalog brighter than this limit may be very compact star clusters.

We visually inspect these two samples of bright objects and remove spurious sources, including obviously saturated foreground stars, hot pixels near chip edges, and the nuclear star cluster. Any objects that overlap between the sample taken from the stellar catalog and the cluster catalog described in Section 2 are also removed. We then attempt to fit the remaining objects from both the cluster and stellar catalogs with GALFIT using the same input parameters as for the observed clusters (see Table 2).

We inspect the residual images of objects that are recovered by GALFIT, meaning their fits successfully converged, and remove objects for which the fit was influenced by the presence of nearby objects. Of the remaining objects for NGC 628c, a total of three objects from the cluster catalog sample were measured to have small radii $\left(r_{\text {eff }} \lesssim 3 \mathrm{pc}\right)$. From the stellar catalog, five objects had reasonable GALFIT fits with a radius $\lesssim 3$ pc. For NGC 628 e, only one object from the cluster catalog had a small radius, and none from the stellar catalog.

For NGC 1313e, there were six objects from the cluster catalog and none from the stellar catalog with small radii. For NGC 1313w, there were four objects from the cluster catalog and none from the stellar catalog with small radii and reasonable GALFIT fits. These results indicate that few compact clusters are likely to have been excluded from the final LEGUS cluster catalogs for NGC 628 and especially for the nearer NGC 1313 system. In addition, given the results from Section 3.3.1, the objects for which GALFIT fits were unsuccessful are unlikely to have $r_{\text {eff }}$ between 1.0 and $3.0 \mathrm{pc}$ in NGC 628, and 0.5 to $3.0 \mathrm{pc}$ in NGC 1313. 


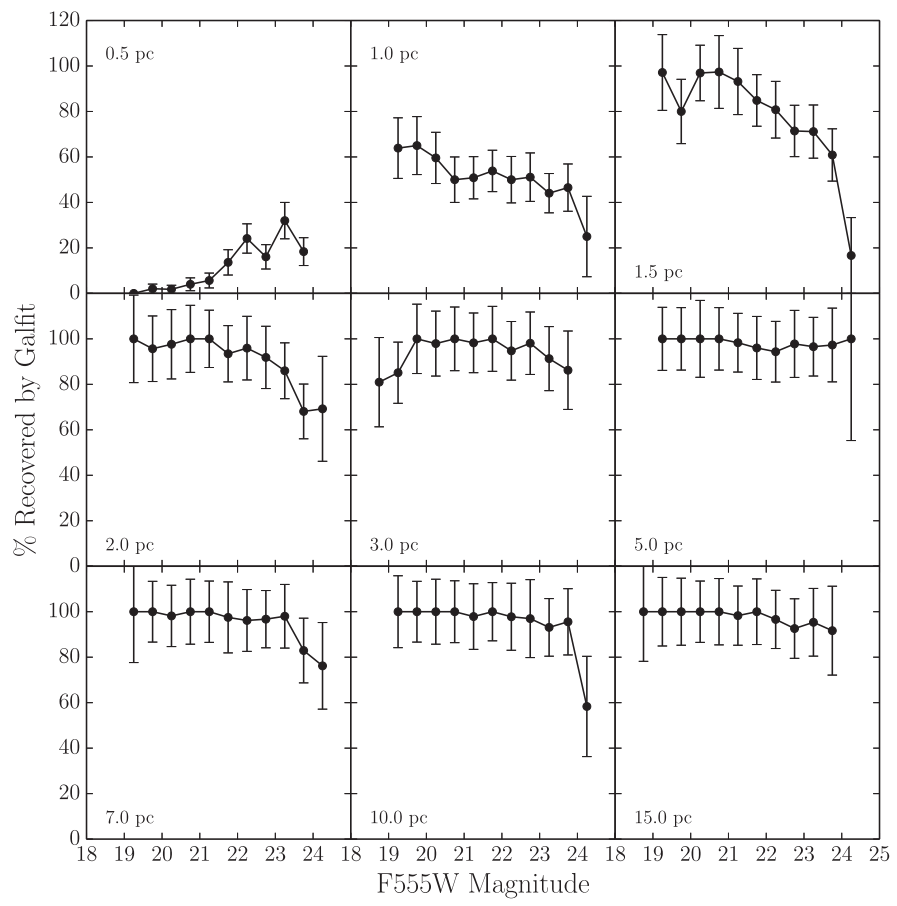

(a) NGC $628 \mathrm{c}$

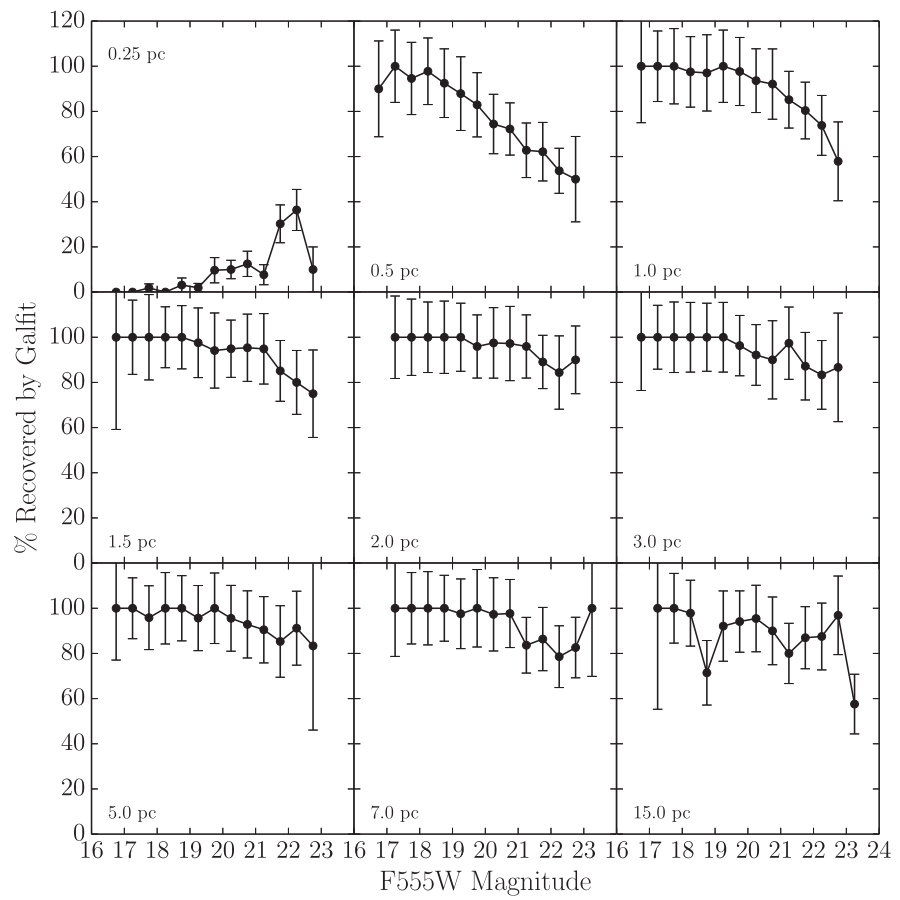

(c) NGC $1313 \mathrm{e}$

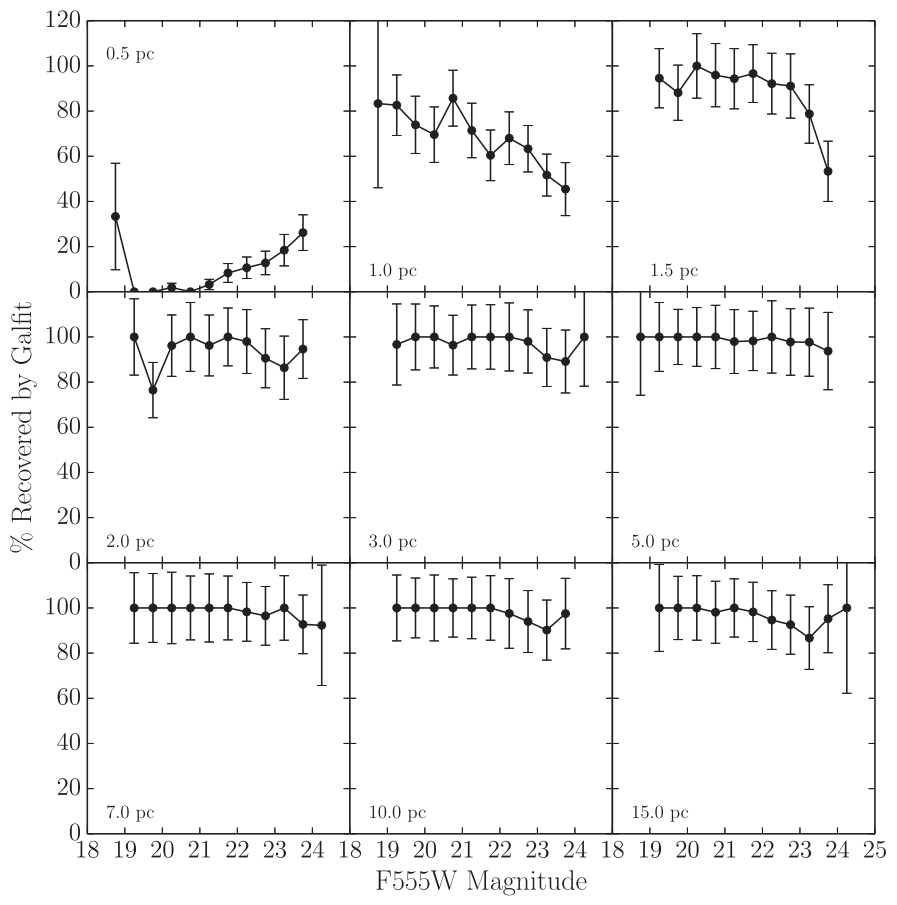

(b) NGC $628 \mathrm{e}$

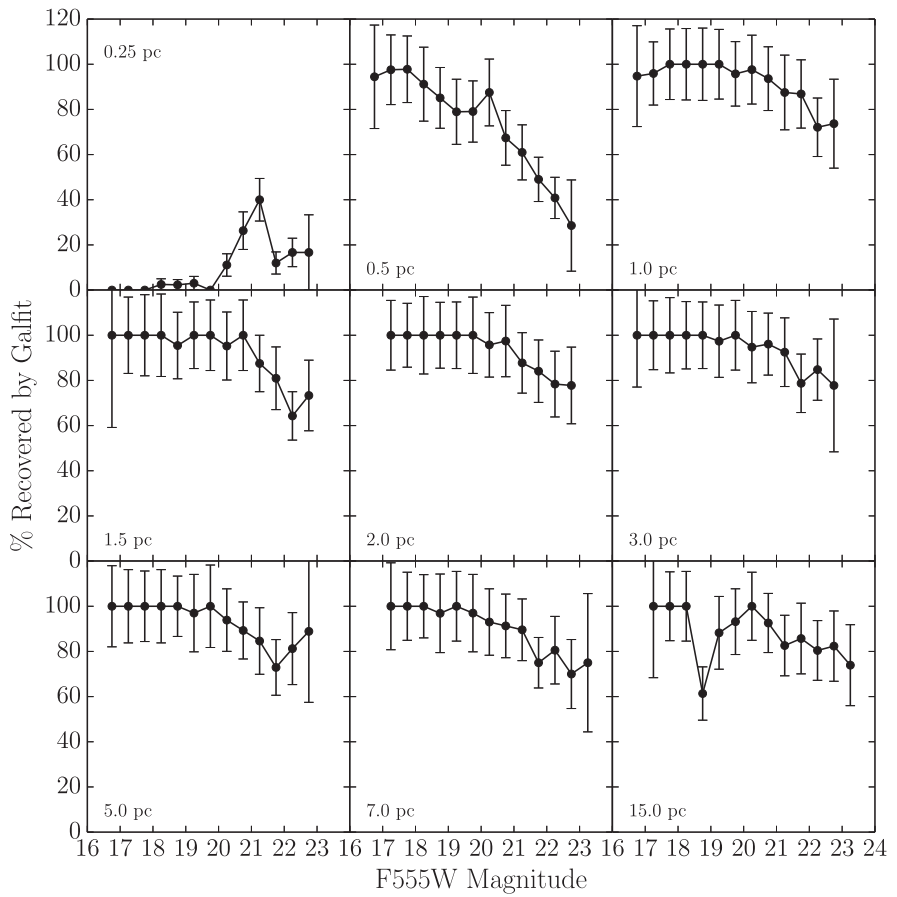

(d) NGC $1313 \mathrm{w}$

Figure 5. Percentage of recovered artificial clusters vs. measured magnitude for each input effective radius for (a) NGC 628c, (b) NGC 628e, (c) NGC 1313e, and (d) NGC 1313w. The low recovered percentages for artificial clusters with sizes of $<0.5 \mathrm{pc}$ in NGC 628 and $<0.25 \mathrm{pc}$ in NGC 1313 are due to resolution effects (see text for details). The error bars represent the Poissonian errors for the number of recovered clusters.

\section{Results}

We present the structural parameters both measured with GALFIT and estimated from CI values for the cluster samples in NGC 628 and NGC 1313 in this section. Tables 3 and 4 contain our measurements for all clusters that satisfy the visual inspection class, age, and mass cuts described in Section 2.
Therefore, there are 320 clusters included in Table 3 for NGC 628 and 195 included in Table 4 for NGC 1313. Those clusters that were successfully fit with GALFIT but for which $\eta \leqslant 1.3$ have very uncertain $r_{\text {eff }}$ (as discussed in Section 4.1), and their $r_{\text {eff }}$ values are enclosed by parentheses in the tables. The astrophysical results that we present are based on these data and thus are subject to inevitable observational selection 
Table 3

Properties of YMCs in NGC 628

\begin{tabular}{|c|c|c|c|c|c|c|c|c|c|}
\hline $\begin{array}{l}\text { Cluster } \\
\text { ID }\end{array}$ & $\begin{array}{l}\text { R.A. } \\
\text { (deg) }\end{array}$ & $\begin{array}{l}\text { Decl. } \\
\text { (deg) }\end{array}$ & $\begin{array}{c}\mathrm{CI} \\
(\mathrm{mag})\end{array}$ & $\eta$ & $\begin{array}{c}\text { GALFIT } \\
\log r_{\text {eff }}(\mathrm{pc})\end{array}$ & $\begin{array}{r}\text { CI-estimated } \\
\log r_{\text {eff }}(\mathrm{pc})\end{array}$ & $\begin{array}{l}\log t_{\text {age }} \\
\text { (years) }\end{array}$ & $\begin{array}{r}\log M \\
\left(M_{\odot}\right)\end{array}$ & $\begin{array}{c}\text { Mode } \\
f_{\text {vis }}\end{array}$ \\
\hline$\overline{196-c}$ & 24.16912 & 15.80396 & 1.649 & $\cdots$ & $\cdots$ & $0.56_{-0.13}^{+0.10}$ & $7.95_{-1.11}^{+0.35}$ & $3.92_{-0.95}^{+0.22}$ & 1.0 \\
\hline $237-c$ & 24.17594 & 15.80297 & 1.650 & $2.18 \pm 0.19$ & $0.38_{-0.08}^{+0.07}$ & $0.56_{-0.02}^{+0.02}$ & $7.04_{-0.00}^{+0.13}$ & $4.11_{-0.00}^{+0.23}$ & 1.0 \\
\hline $256-c$ & 24.17687 & 15.80270 & 1.491 & $\cdots$ & $\ldots$ & $0.29_{-0.17}^{+0.12}$ & $8.30_{-0.30}^{+0.00}$ & $3.72_{-0.16}^{+0.07}$ & 1.0 \\
\hline $268-c$ & 24.16487 & 15.80224 & 1.664 & $\cdots$ & $\ldots$ & $0.58_{-0.02}^{+0.02}$ & $7.48_{-0.00}^{+0.00}$ & $4.37_{-0.03}^{+0.01}$ & 2.0 \\
\hline $292-c$ & 24.17258 & 15.80185 & 1.722 & $\cdots$ & $\cdots$ & $0.65_{-0.12}^{+0.09}$ & $8.00_{-1.15}^{+0.30}$ & $3.93_{-0.94}^{+0.23}$ & 2.0 \\
\hline
\end{tabular}

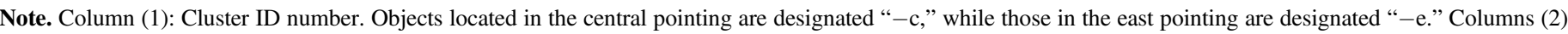

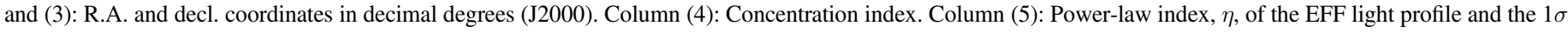

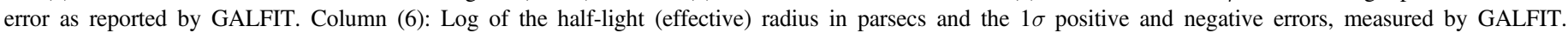

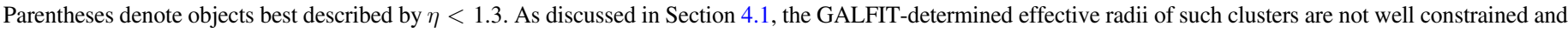

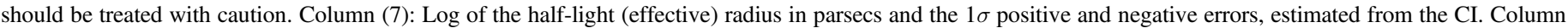

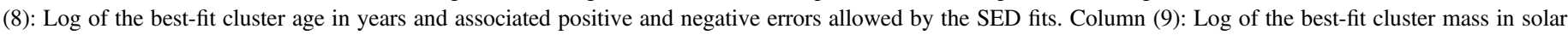
masses and associated positive and negative errors allowed by the SED fits. Column (10): LEGUS visual inspection class, mode.

(This table is available in its entirety in machine-readable form.)

effects. However, the sample for this study was carefully selected to include star clusters that are likely to be bound and sufficiently massive so as to minimize the effects of stochastic sampling of their stellar populations. Thus this study includes what is perhaps the most complete sample of relatively massive $\left(M>5000 M_{\odot}\right)$, young star clusters for which structural measurements have yet been obtained.

\subsection{Distribution of Effective Radii}

As discussed in detail in Paper I (see Section 4.1 and Figure 1 in that work), when GALFIT finds that a cluster's light profile slope is very shallow, $\eta \approx 1.0$, the effective radius we calculate is very uncertain. To avoid unphysical radii and errors, we restrict our analysis of the GALFIT samples to those objects with $\eta \geqslant 1.3$. The number of clusters remaining in the GALFIT samples after imposing this limit on $\eta$ is 134 in NGC 628 (107 in the central pointing and 27 in the east pointing) and 59 in NGC 1313 (14 in the east pointing and 45 in the west pointing). This cut on the GALFIT sample naturally introduces a bias against clusters with shallow radial intensity profiles, which is why we also include results from the CI method. However, for clusters where we could obtain models with GALFIT, we expect the results to be more robust.

We plot the distributions of effective radii for NGC 628 and NGC 1313 in Figure 6. The panels in the top and middle rows show the clusters located in the two pointings on each galaxy separately. The panels in the bottom row show the combination of the two pointings for each galaxy. The effective radii measured from GALFIT fits are plotted as blue histograms, while the CIestimated effective radii are plotted as orange histograms. The widths of the bins were estimated using the Freedman-Diaconis rule (Ivezić et al. 2014) and rounded to the nearest tenth for each panel. The blue and orange curves show the result of representing each cluster as a Gaussian kernel and summing the kernels together.

Each Gaussian kernel is centered on the radius of a cluster, has a bandwidth (standard deviation) equal to the error on the radius of the cluster, and is normalized by the number of clusters in the sample. ${ }^{17}$ The noisy appearance of these curves

\footnotetext{
17 This is a slight variation on the nonparametric statistical method known as kernel density estimation (Ivezić et al. 2014), which is intended to give an accurate representation of the shape of the underlying distribution without using a histogram, which assumes bin width and placement.
}

in the NGC 1313 panels is due to the relatively small effective radius errors for some of the clusters, which results in narrow Gaussian kernels. We believe this may be the result of a much lower uncertainty in the distance to NGC 1313 as compared to NGC 628, which is combined with the FWHM and $\eta$ uncertainties to determine the effective radius errors. The CI limits listed in Table 1 for each pointing have been converted to effective radii using the relation between CI and effective radius determined in Section 3.2 and are plotted as vertical dashed lines.

In each individual panel, the distributions of GALFITmeasured radii and CI-estimated radii are very similar in shape, peak location, and overall extent. We perform AndersonDarling tests to determine if the distributions in each panel are significantly different from each other. This takes the form of rejecting or accepting the null hypothesis, which is that the distributions in each panel are consistent with being drawn from the same parent distribution. In all panels except for NGC 628c, we cannot reject the null hypothesis $(p>0.19$ for all five panels), meaning that the GALFIT-measured radii and CI-estimated radii are consistent with being drawn from the same parent population. For NGC 628c, we can reject the null hypothesis at a significance level of $7 \%(p=0.07)$, suggesting that the two distributions in this panel are somewhat different from each other. This probably is due to the offset of the CI sample toward larger radii by a small but statistically significant amount.

The median effective radii are listed in Table 5 for each pointing separately and for the combined sample from each galaxy. We find that the two methods for estimating effective radii produce very similar medians and MADs, which gives confidence in the CI-estimated effective radius technique. This technique can therefore be used to derive effective radii from much larger samples of clusters than is possible with our GALFIT method.

Comparing NGC 628 to NGC 1313, the overall shapes of the effective radius distributions are relatively similar and also resemble the distribution presented in Paper I for M83 (see Figure 2 in that work). To a first approximation, the distributions of $r_{\mathrm{eff}}$ in M83, NGC 628, and NGC 1313 have lognormal shapes and peak at $\sim 3$ pc. We perform AndersonDarling tests to statistically compare the GALFIT-measured and CI-estimated radius distributions for NGC 628 and 
Table 4

Properties of YMCs in NGC 1313

\begin{tabular}{|c|c|c|c|c|c|c|c|c|c|}
\hline $\begin{array}{l}\text { Cluster } \\
\text { ID }\end{array}$ & $\begin{array}{l}\text { R.A. } \\
\text { (deg) }\end{array}$ & $\begin{array}{l}\text { Decl. } \\
(\mathrm{deg})\end{array}$ & $\begin{array}{c}\mathrm{CI} \\
(\mathrm{mag})\end{array}$ & $\eta$ & $\begin{array}{r}\text { GALFIT } \\
\log r_{\text {eff }}(\mathrm{pc})\end{array}$ & $\begin{array}{r}\text { CI-estimated } \\
\log r_{\text {eff }}(\mathrm{pc})\end{array}$ & $\begin{array}{l}\log t_{\text {age }} \\
\text { (years) }\end{array}$ & $\begin{array}{l}\log M \\
\left(M_{\odot}\right)\end{array}$ & $\begin{array}{c}\text { Mode } \\
f_{\text {vis }}\end{array}$ \\
\hline 45 -e & 49.61172 & -66.45683 & 2.007 & $1.02 \pm 0.05$ & $\left(8.00_{-8.00}^{+1.64}\right)$ & $0.67_{-0.04}^{+0.04}$ & $8.30_{-0.00}^{+0.00}$ & $4.72_{-0.00}^{+0.03}$ & 1.0 \\
\hline $108-\mathrm{e}$ & 49.64161 & -66.46315 & 1.871 & $1.01 \pm 0.08$ & $\left(15.36_{-15.36}^{+2.44}\right)$ & $0.49_{-0.05}^{+0.05}$ & $8.00_{-0.22}^{+0.00}$ & $4.03_{-0.09}^{+0.04}$ & 2.0 \\
\hline $133-\mathrm{e}$ & 49.57730 & -66.46528 & 1.866 & $1.03 \pm 0.07$ & $\left(5.16_{-5.16}^{+1.44}\right)$ & $0.48_{-0.03}^{+0.03}$ & $8.30_{-0.00}^{+0.00}$ & $3.99_{-0.04}^{+0.06}$ & 2.0 \\
\hline $215-e$ & 49.60231 & -66.46820 & 1.652 & $1.16 \pm 0.06$ & $\left(0.70_{-0.56}^{+0.24}\right)$ & $0.14_{-0.02}^{+0.02}$ & $7.48_{-0.36}^{+0.00}$ & $3.86_{-0.32}^{+0.05}$ & 1.0 \\
\hline $228-\mathrm{e}$ & 49.57860 & -66.46854 & 1.635 & $1.01 \pm 0.03$ & $\left(14.74_{-14.74}^{+2.02}\right)$ & $0.11_{-0.03}^{+0.03}$ & $8.30_{-0.00}^{+0.00}$ & $3.98_{-0.04}^{+0.04}$ & 2.0 \\
\hline
\end{tabular}

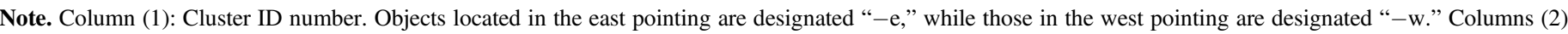

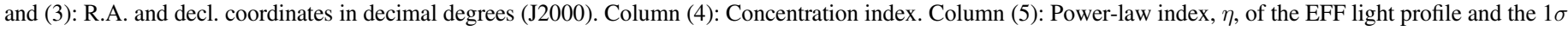

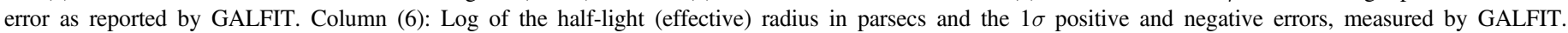

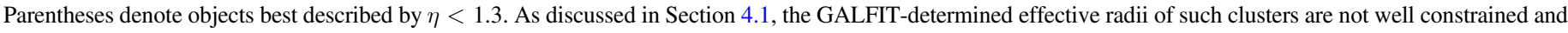

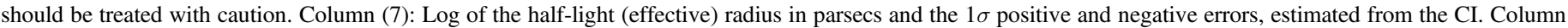

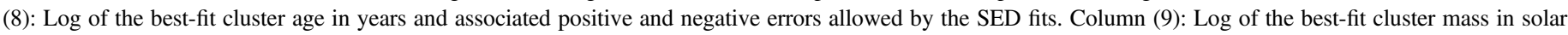
masses and associated positive and negative errors allowed by the SED fits. Column (10): LEGUS visual inspection class, mode.

(This table is available in its entirety in machine-readable form.)

NGC 1313, and we find that we can reject the null hypothesis at high significance for both methods of measurement ( $p=0.001$ for GALFIT and $p=1.3 \times 10^{-5}$ for CI). Therefore, despite their approximate similarities, the distributions of effective radii in these two galaxies are formally inconsistent with being drawn from the same parent population.

We suggest that this difference is an observational artifact because in NGC 628, the smallest cluster radius we measure is about 1 pc, whereas in NGC 1313 (and M83 as well), we find a tail in the distribution to small radii, $\sim 0.3$ pc. Because NGC 628 is about twice as distant as NGC 1313 and M83, the $\mathrm{CI}$ limit for sample selection corresponds to a larger radius and therefore likely results in the smallest clusters being removed from that sample. From Figure 5, at least $40 \%$ of artificial clusters with a radius of $1 \mathrm{pc}$ are lost at all magnitudes in NGC 628, whereas for NGC 1313, all input clusters at this radius are well recovered. Therefore, we conclude that the physical distributions of cluster $r_{\text {eff }}$ are similar to each other, at least when considering the parameter space covered by this study.

In all three galaxies, the largest clusters for which $\eta \geqslant 1.3$ are about $10 \mathrm{pc}$ in radius. We do not expect to find very large clusters in the GALFIT-measured samples with this conservative $\eta$ cut, and we also do not find very large clusters in the CI-estimated radius distributions in Figure 6. However, we note that the CI method of estimating $r_{\text {eff }}$ becomes less sensitive as the CI increases; that is, a small uncertainty in CI leads to a big uncertainty in $r_{\text {eff }}$ for large $r_{\text {eff }}$. An extended tail of clusters with large radii appears in the M83 distribution when clusters with shallower light profiles are included $(\eta \geqslant 1.1$, see bottom panel of Figure 2 in Paper I). Therefore, the properties and numbers of clusters with shallow profiles remain uncertain.

Several studies of YMCs and GCs in the Milky Way and other nearby galaxies have found effective radius distributions strikingly similar to those we present here and in Paper I (e.g., Larsen 2004; Scheepmaker et al. 2007; Barmby et al. 2009; Bastian et al. 2012; Puzia et al. 2014). In particular, the location of the peak in the observed distribution of $r_{\text {eff }}$ appears to be quite robust and is consistently found to be at 2-3 pc across a range of cluster mass, age, and environment. We find that NGC 628 and NGC 1313 provide more evidence in support of this conclusion, especially given the results of our completeness tests in Section 3.3 and the fact that the CI limits are located significantly below the peaks of the effective radius distributions.

\subsection{Effective Radius as a Function of Cluster Properties}

In Figure 7, we plot effective radius as a function of age for the clusters in NGC 628 (left column) and NGC 1313 (right column). We combine the cluster samples from the individual pointings into one for each galaxy since their properties were not found to be significantly different in Figure 6 . The top panels show the effective radii measured with GALFIT, and the bottom panels show the CI-estimated effective radii. In each panel, we plot the median effective radii in equal-size bins in $\log \left(t_{\text {age }}\right)$ space (orange lines) and in bins containing equal numbers of clusters (green lines). Each bin contains at least five clusters. The dashed lines of each color show the 16th and 84th percentiles in radius for each bin.

We calculate Kendall's $\tau$, a nonparametric correlation coefficient, to determine if the effective radii and cluster ages are correlated in each panel (see the Appendix for a short discussion of the definition and applicability of Kendall's $\tau$ ). We find modest correlations in both panels for NGC 628 with high significance. As labeled in the figure, Kendall's $\tau$ statistic is $\sim 0.25$ for both panels, and the associated $p$ values are $p=6.5 \times 10^{-6}$ for the GALFIT sample and $p=1.3 \times 10^{-10}$ for the CI sample. Because these $p$ values are smaller than our selected significance level of $p=2.7 \times 10^{-3}$ (corresponding to a $3 \sigma$ significance level for a two-tailed test), we can reject the null hypothesis of the Kendall's $\tau$ correlation test, which means we confirm statistically significant correlations for both panels.

Assuming a power-law relation exists between cluster radius and age, we perform simple least-squares fits to the median effective radii from each binning procedure, including standard errors on the medians, and find very shallow slopes of $\sim 0.1$. For NGC 1313, we find slightly stronger correlations, also with high significance. For the GALFIT sample, $\tau \sim 0.28$ and $p=1.6 \times 10^{-3}$, while $\tau \sim 0.33$ and $p=4.3 \times 10^{-12}$ for the CI-estimated sample. The least-squares fits result in slopes of $\sim 0.3$, which agrees with the trend found in Figure 3 in Paper I.

We note that the smaller sample size for NGC 1313 may lead to larger scatter and increased uncertainty in the radiusage relation than for NGC 628 . However, the cluster samples in NGC 1313 and M83 extend to smaller radii than NGC 628, and the strength of the radius-age relation for these two 


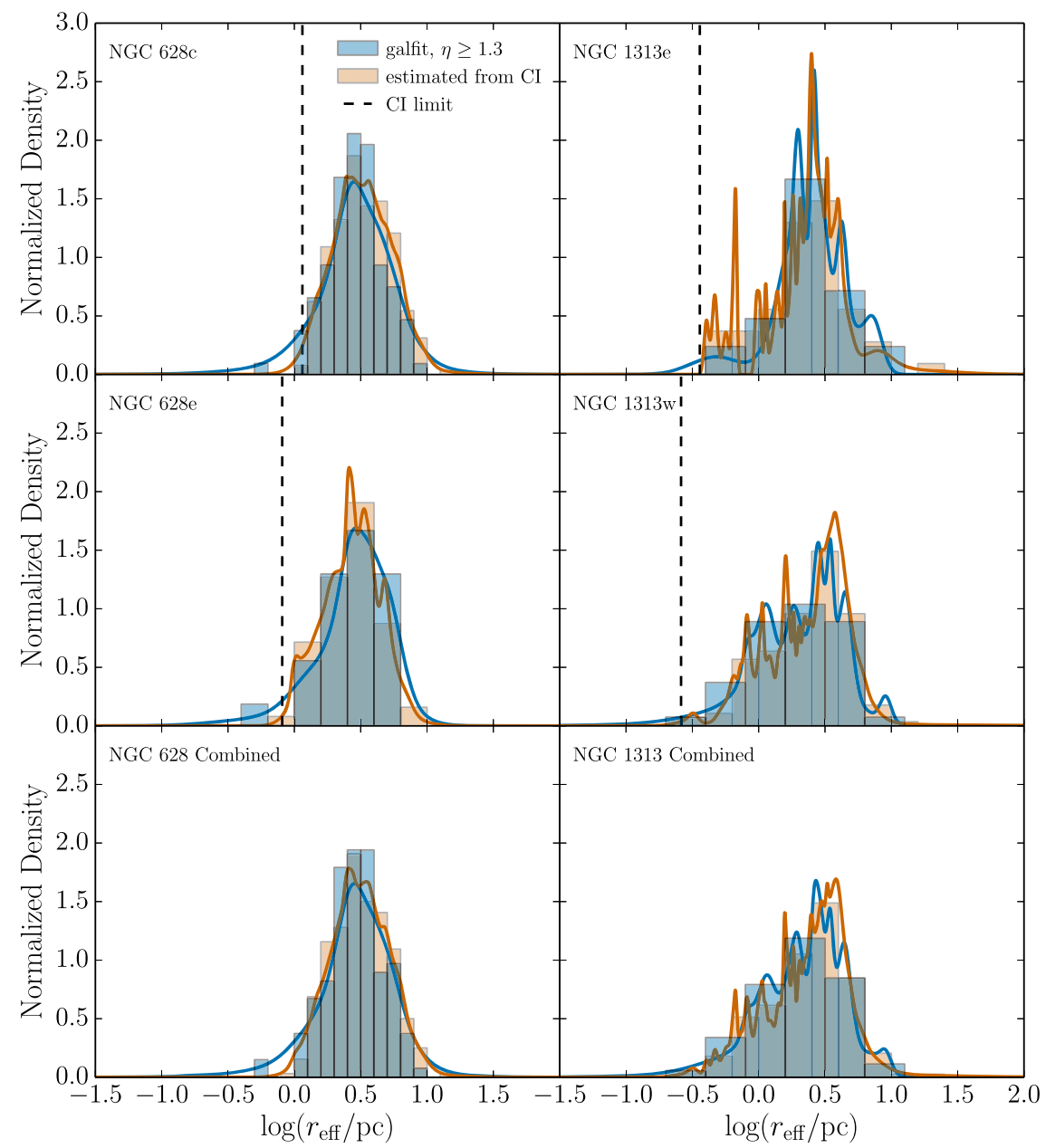

Figure 6. Distributions of effective radii for NGC 628 (left column) and NGC 1313 (right column) clusters. The top two panels in each column show the radius distributions for the individual pointings, and the bottom panels show the pointings on each galaxy combined. Effective radii from GALFIT fits are plotted as blue histograms, and CI-estimated effective radii are plotted as orange histograms. The solid curves show summed Gaussian kernels with widths equal to the error on each radius measurement. The vertical dashed lines show the location of the CI limit imposed on the LEGUS cluster catalog.

Table 5

Median Effective Radii

\begin{tabular}{|c|c|c|c|c|}
\hline $\begin{array}{l}\text { LEGUS } \\
\text { Pointing }\end{array}$ & $\begin{array}{l}\text { GALFIT Median } \\
\quad r_{\text {eff }}(\mathrm{pc})\end{array}$ & $\begin{array}{l}\text { CI Median } \\
r_{\text {eff }}(\mathrm{pc})\end{array}$ & $\begin{array}{c}\text { GALFIT } \\
N_{\text {clusters }}\end{array}$ & $\begin{array}{c}\text { CI } \\
N_{\text {clusters }}\end{array}$ \\
\hline NGC 628c & $2.9 \pm 0.9$ & $3.2 \pm 1.1$ & 107 & 257 \\
\hline NGC $628 \mathrm{e}$ & $2.9 \pm 1.0$ & $2.7 \pm 0.9$ & 27 & 63 \\
\hline NGC 628 all & $2.9 \pm 0.9$ & $3.1 \pm 1.1$ & 134 & 320 \\
\hline NGC $1313 \mathrm{e}$ & $2.7 \pm 1.0$ & $2.4 \pm 0.9$ & 14 & 54 \\
\hline NGC 1313w & $2.0 \pm 1.0$ & $2.8 \pm 1.2$ & 45 & 141 \\
\hline NGC 1313 all & $2.3 \pm 1.2$ & $2.7 \pm 1.1$ & 59 & 195 \\
\hline
\end{tabular}

galaxies is greater than for NGC 628. The proximity of both NGC 1313 and M83 ( 4 Mpc) has allowed us to detect smaller clusters in those galaxies as compared to NGC 628 and perhaps has provided better leverage on the radius-age relation. As discussed in Paper I, a few studies have found slight positive or negative correlations between radius and age, while others have found essentially no correlation at all (e.g., Larsen 2004; McLaughlin \& van der Marel 2005; Scheepmaker et al. 2007; Bastian et al. 2012). The positive correlations presented here and in Paper I suggest that, on average, YMCs expand slightly over the first few hundred megayears of evolution.

In Figure 8, we plot effective radius as a function of distance from the center of the galaxy for the clusters in NGC 628 (left column) and NGC 1313 (right column). Again we combine the cluster samples from the individual pointings for each galaxy, and the top panels show the radii measured with GALFIT, while the bottom panels show the CI-estimated radii. The solid and dashed lines are the same as in Figure 7 and show running medians and $1 \sigma$ percentiles in effective radius for different binning techniques.

The clusters in NGC 628 are located between $\sim 300 \mathrm{pc}$ and $10 \mathrm{kpc}$ from its center, while the clusters in NGC 1313 extend closer to the galactic center, the closest being about $30 \mathrm{pc}$ from the center. No correlation between cluster radius and galactocentric distance is apparent to the eye. We again calculate Kendall's $\tau$ to determine if a correlation is present, and we find no statistically significant correlations in any of the four panels of Figure 8. We divide the samples in both galaxies into three bins in cluster age, $<50 \mathrm{Myr}, 50-100 \mathrm{Myr}$, and 100-200 Myr, chosen to reflect the age ranges over which the mean cluster mass is relatively constant (especially for NGC 628), as shown in Figure 3. Dividing the samples into bins in age also minimizes any effect of cluster expansion with time. We again find no statistically significant correlations with the Kendall's $\tau$ correlation test in any of the bins in cluster age.

We are able to probe a larger range of galactocentric distance with these data than with the M83 sample, but no significant correlation is found in any of the three galaxies. Again, the 


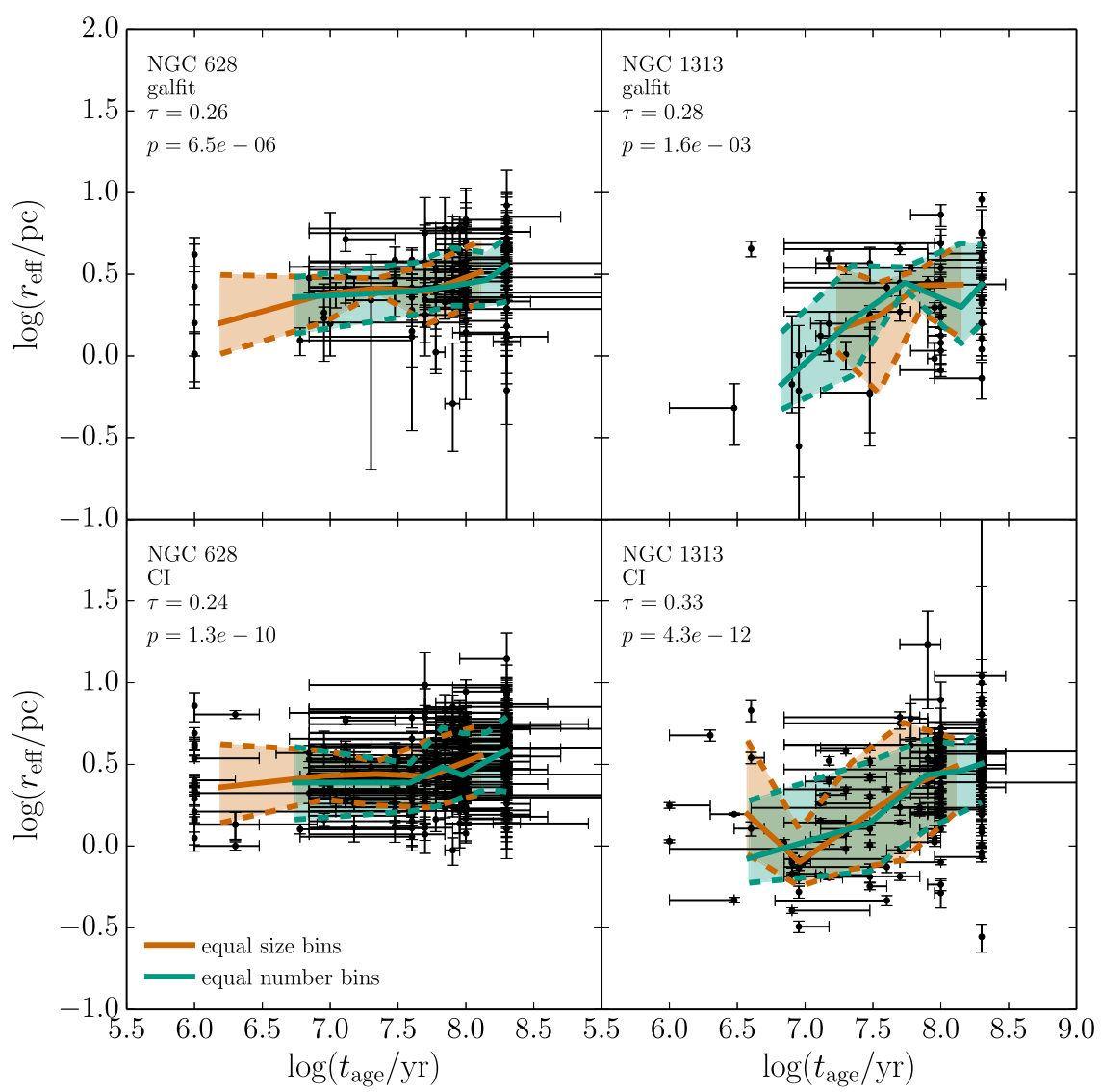

Figure 7. Effective radius as a function of cluster age for NGC 628 (left column) and NGC 1313 (right column). The top row of panels shows the effective radii measured with GALFIT, and the bottom row shows those estimated from CI values. The solid lines show median effective radii in equal-size bins in log space (orange) and in bins with equal numbers of clusters (green). The dashed lines and shaded areas show the extent of the 16th and 84th percentiles in radius for each binning method. The Kendall $\tau$ correlation statistic and associated $p$ value are located in the top left corner of each panel.

evidence from other studies is mixed: some find slight correlations between YMC radius and distance, and others find none (e.g., Bastian et al. 2005, 2012; Scheepmaker et al. 2007; Barmby et al. 2009; Sun et al. 2016). The sizes of GCs appear to be shallowly related to their galactocentric distances, though in some cases, like the Milky Way, the relationship is stronger (e.g., van den Bergh et al. 1991; Harris 2009; Harris et al. 2010; Masters et al. 2010).

Models where young star clusters quickly fill their tidal radii are attractive. But in this case the radii of star clusters should eventually reflect their local tidal gravitational fields that usually decrease with increasing galactocentric radius. Our results suggest that clusters across the range of mass, age, and environment covered by our samples do not show effects of tidal truncations in their half-light radii; otherwise, a stronger increase of cluster size with galactocentric distance is expected (e.g., Gieles et al. 2011; Madrid et al. 2012; Webb et al. 2016).

In Figure 9, we plot effective radius as a function of cluster mass for the clusters in NGC 628 (left column) and NGC 1313 (right column). Again, the cluster samples from the individual pointings on each galaxy are combined, and the top panels show the radii measured with GALFIT, while the bottom panels show the CI-estimated radii. The solid and dashed lines are the same as in Figure 7. No strong correlation is obvious in the NGC 628 panels, and none is present according to the Kendall's $\tau$ correlation test. In NGC 1313, however, we find statistically significant trends in both the GALFIT $(\tau=0.3$ and $\left.p=6.6 \times 10^{-4}\right)$ and CI-based samples $(\tau=0.2$ and $p=5.7 \times 10^{-4}$ ). This may be another indication of the importance of including clusters with small $r_{\text {eff }}$, which tend to be young and have lower masses. Assuming a power-law relation between cluster radius and mass, we perform simple least-squares fits to the median effective radii from both binning techniques, including standard errors on the medians. We find steeper power-law slopes for the GALFIT sample, $\sim 0.5$, than for the CI-estimated sample, $\sim 0.2-0.3$.

We further investigate the correlation found in Figure 9 for NGC 1313 by dividing the sample into three bins in cluster age $(<50 \mathrm{Myr}$, 50-100 Myr, and 100-200 Myr) and plotting the results in Figure 10. Again, the GALFIT sample is plotted in the left column and the CI-estimated sample in the right column. Although correlations are apparent to the eye, by calculating Kendall's $\tau$ for each panel, we find no significant correlations between effective radius and cluster mass. Perhaps the radius-age relation for NGC 1313 drives the correlation found in Figure 9 because more massive clusters have a higher likelihood of being older, statistically (see Figure 3). Alternatively, the small numbers of clusters in each age bin may prevent a statistically significant correlation from being found. In addition, it is possible our cluster samples do not extend to old enough ages to detect a correlation between effective radius and cluster mass, as found in the oldest bin in Paper I.

Previous studies of clusters below $\sim 10^{6} M_{\odot}$ have found little to no correlation between effective radius and cluster mass (e.g., Larsen 2004; Bastian et al. 2005, 2012; Scheepmaker et al. 2007; Barmby et al. 2009). Above $\sim 10^{6} M_{\odot}$, the radii of 


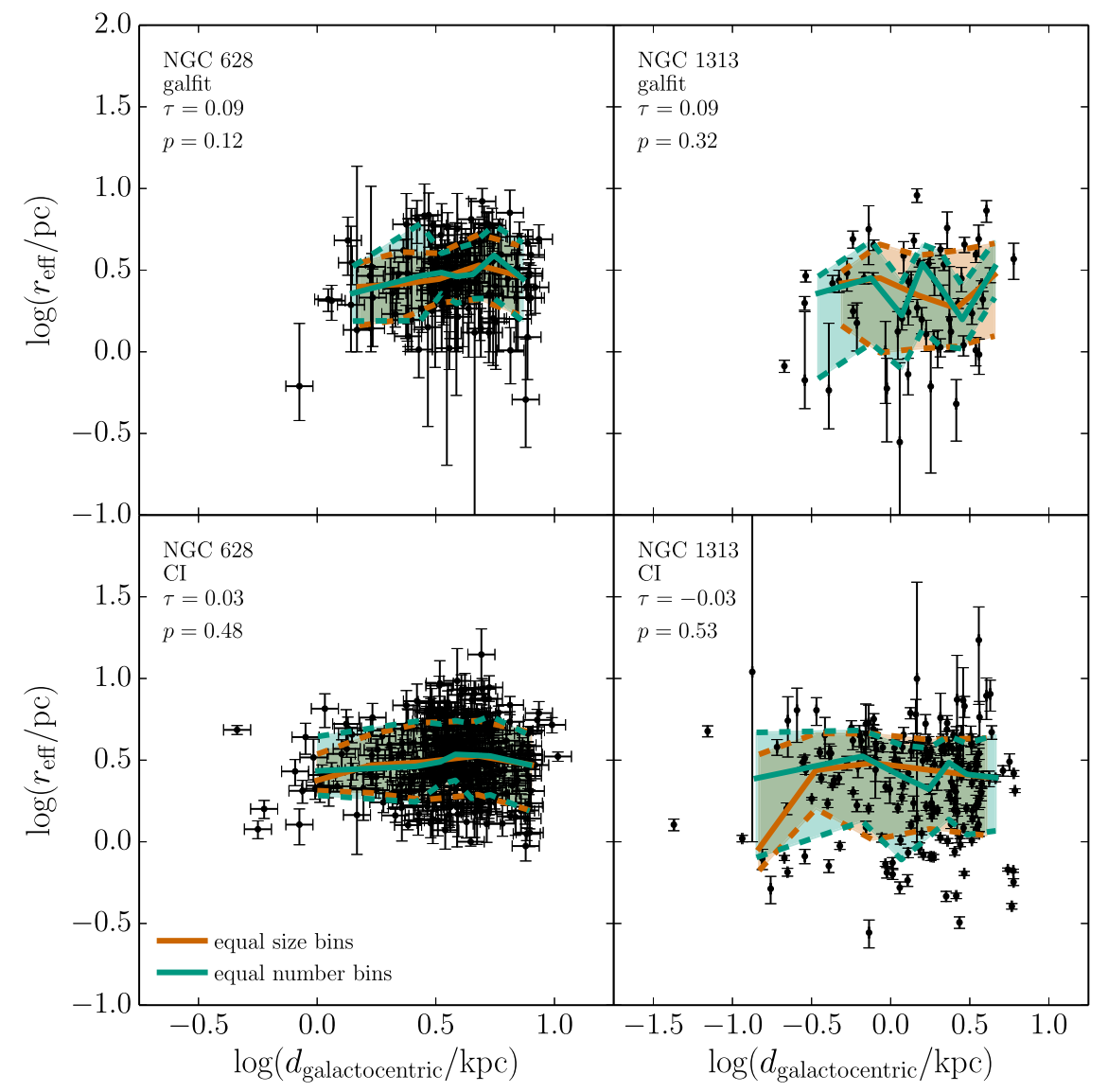

Figure 8. Effective radius as a function of galactocentric distance for NGC 628 (left column) and NGC 1313 (right column). The effective radii measured with GALFIT and estimated from CI values are located in the top and bottom rows of panels, respectively. The solid lines, dashed lines, and shaded regions are the same as in Figure 7. The Kendall $\tau$ correlation statistic and associated $p$ value are given in each panel. Note the different scales on the $x$ axis for NGC 628 vs. NGC 1313 .

both YMCs and GCs appear to increase with mass (or luminosity; e.g., Kissler-Patig et al. 2006; Harris 2009; Fall \& Chandar 2012; Bastian et al. 2013).

\subsection{EFF Profile Indices}

The power-law index of the EFF light profile, $\eta$, describes the slope of the wings of a star cluster's intensity profile. We find that the ranges of light profile indices of the clusters fit by GALFIT are $1.0 \leqslant \eta \leqslant 9.3$ in NGC 628 and $1.0 \leqslant \eta \leqslant 5.6$ in NGC 1313, though the majority of clusters in both galaxies are best described by $1.0 \leqslant \eta \leqslant 3.0$. The median $\eta$ values are 1.4 for NGC 628 and 1.2 for NGC 1313. The smallest possible value of $\eta$ is artificially set to 1.0 by GALFIT because a light profile with $\eta<1.0$ must, by definition, contain an infinite amount of light. However, $\eta$ values smaller than 1.0 have been recovered for other clusters using different techniques (Larsen 2004). The median $\eta$ values we find could therefore be smaller in reality. In any case, the range of $\eta$ values we find agrees with those presented in Paper I and several other studies of cluster radial intensity profiles in nearby galaxies (e.g., Elson et al. 1987; Mackey \& Gilmore 2003; Glatt et al. 2009).

We also look for any relationships between $\eta$ and cluster age, mass, and galactocentric distance, similarly to Section 4.2. We perform Kendall's $\tau$ correlation tests and find no statistically significant correlations between $\eta$ and any cluster property. This agrees with our findings for M83 in Paper I. Therefore, we do not find evidence to support the increase in $\eta$ with age as reported by Larsen (2004), which may indicate that the light profiles of YMCs are rather robust during their early evolution.

\subsection{Dynamical Age}

We also calculate the dynamical age, $\Pi$, as defined by Gieles \& Portegies Zwart (2011), for our objects. The dynamical age $\Pi$ is the ratio of cluster age to the crossing time, $\Pi \equiv t_{\text {age }} / t_{\text {cross }}$, and can be used to determine if a system is gravitationally bound at the present time. We use Equation (1) from Gieles \& Portegies Zwart (2011) to calculate crossing times with the measured effective radii and masses. If the age of a stellar system exceeds its current crossing time, $\Pi>1$, then the stars in that system have remained clustered together in space for their lifetimes and have not freely expanded into their surroundings, which implies that the system is likely gravitationally bound. As discussed in Gieles \& Portegies Zwart (2011), the assumption of virial equilibrium in Equation (1) overestimates the crossing time of unbound associations as the objects age and expand freely. Therefore, for older associations, $\Pi$ is underestimated, aiding in the distinction between bound and unbound objects. Of course, the true dynamical state of a stellar system cannot be determined without complete kinematical measurements of the constituent stars, but such measurements are nontrivial and beyond the scope of this work. Despite this limitation, Portegies Zwart et al. (2010) and Bastian et al. (2012) have found the dynamical age, $\Pi$, to be a useful discriminator between bound clusters and unbound stellar associations. 


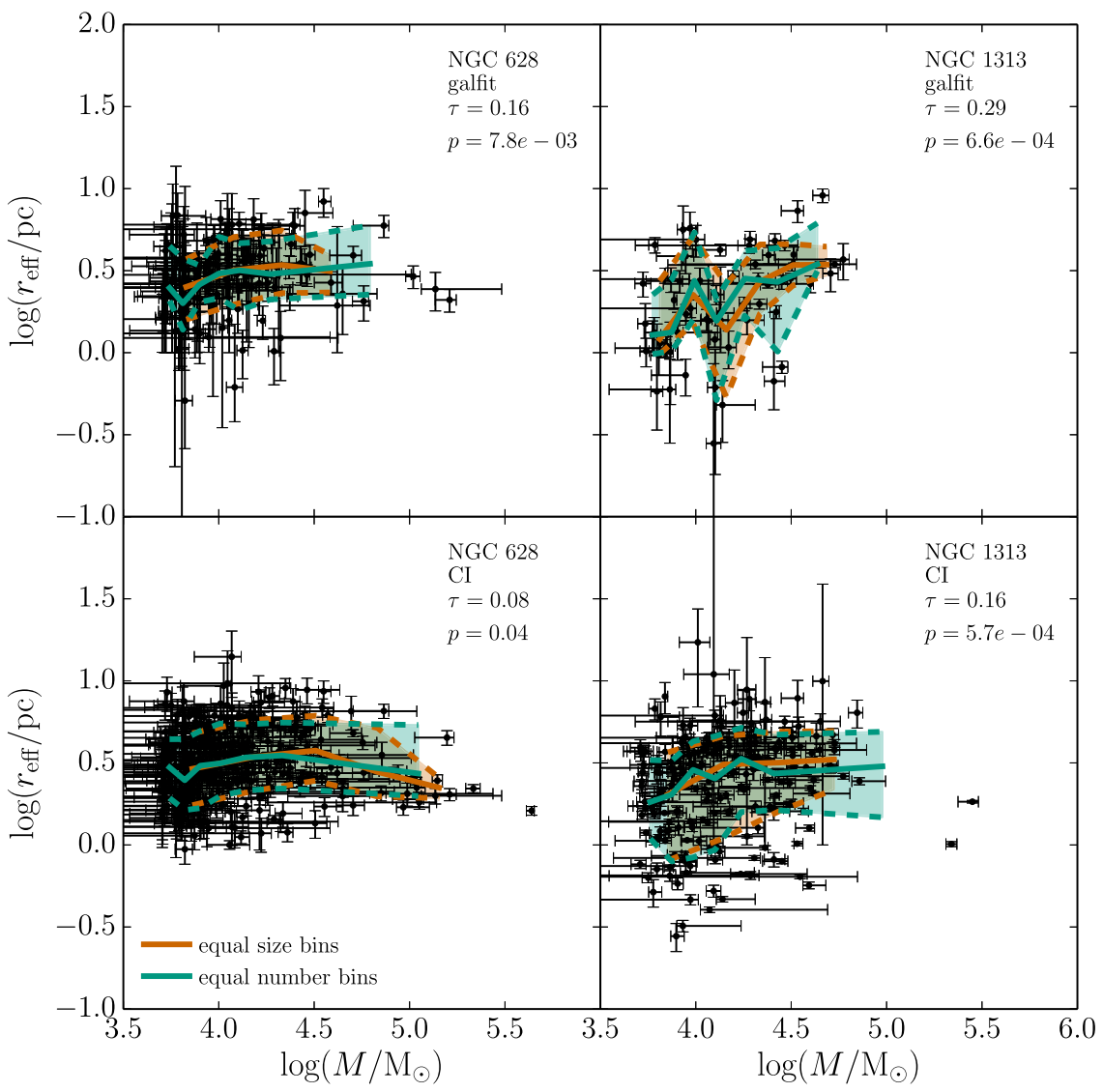

Figure 9. Effective radius as a function of cluster mass for NGC 628 (left column) and NGC 1313 (right column). The top row of panels shows the effective radii measured with GALFIT, and the bottom row shows those estimated from CI values. The solid lines, dashed lines, and shaded regions are the same as in Figure 7. The Kendall $\tau$ correlation statistic and associated $p$ value are given in each panel.

In Figure 11, we present the distribution of dynamical ages of the clusters in NGC 628 (left panel) and NGC 1313 (right panel). The blue (darker) histograms correspond to dynamical ages calculated with effective radii determined from GALFIT, and the orange (lighter) histograms correspond to those using CI-estimated effective radii. The black dashed lines separate the bound and unbound objects according to $\Pi$. The peak in the $\Pi$ distribution (particularly for NGC 628) is a selection effect and is a consequence of the clusters having a roughly constant radius and an age limit of $200 \mathrm{Myr}$.

Clearly, the vast majority of objects in both galaxies fall in the region $\Pi>1$, which means they are most likely bound systems. In NGC 628, only $4 \%$ of objects in the GALFIT sample and $8 \%$ of the objects in the CI-estimated sample can be classified as unbound. In NGC 1313, those percentages are 4\% and $2 \%$, respectively. Essentially all of the objects for which $\Pi<1$ are quite young, $\lesssim 10 \mathrm{Myr}$, so the dynamical state of these few objects is ambiguous, as noted in Gieles \& Portegies Zwart (2011). However, it appears that the LEGUS selection criteria and visual inspection techniques successfully produce samples of (likely) gravitationally bound star clusters, as intended (Adamo et al. 2017).

\subsection{Jacobi Radii}

The Jacobi radius of a cluster can help to determine if the tidal field of the host galaxy has a strong influence on the evolution of the cluster. It sets the size of the zero-velocity surface of a cluster in a tidal field and therefore also defines the volume over which stars are bound to the cluster. If the ratio of the half-mass radius (about $4 / 3 \times r_{\text {eff }}$ ) to the Jacobi radius is about 0.15 or 0.2 , the cluster is filling its Roche volume, and its evolution will be affected by the galaxy's tidal field (Hénon 1961; Alexander et al. 2014). A cluster with a halfmass to Jacobi radius ratio less than 0.15 or 0.2 will evolve as essentially an isolated system and expand gradually. A tidally filling cluster should contract as it loses mass (Heggie \& Hut 2003).

For a galaxy with a flat rotation curve and a nonrotating cluster, the Jacobi radius is defined by Equation (9) in Portegies Zwart et al. (2010):

$$
r_{\mathrm{J}}=\left(\frac{G M}{2 \omega^{2}}\right)^{1 / 3},
$$

which requires knowledge of the cluster mass, $M$, and the angular speed of the galaxy's rotation, $\omega$. According to $\mathrm{H} \alpha$ kinematics from Daigle et al. (2006), NGC 628 has a flat rotation curve with a deprojected rotation velocity of about $175 \mathrm{~km} \mathrm{~s}^{-1}$ beyond $\sim 2.4 \mathrm{kpc}$ in the galaxy's disk. NGC 1313, on the other hand, has a $\mathrm{HI}$ rotation curve that is increasing linearly within $\sim 7.5 \mathrm{kpc}$, and the behavior beyond this distance is unclear (Ryder et al. 1995). Because Equation (3) assumes a flat rotation curve, we can only estimate Jacobi radii for clusters in NGC 628 at this time. There are few observational studies of the rotation of young clusters themselves, and those that do exist find that the rotational energy of such objects is relatively small (e.g., Hénault-Brunet et al. 2012). 


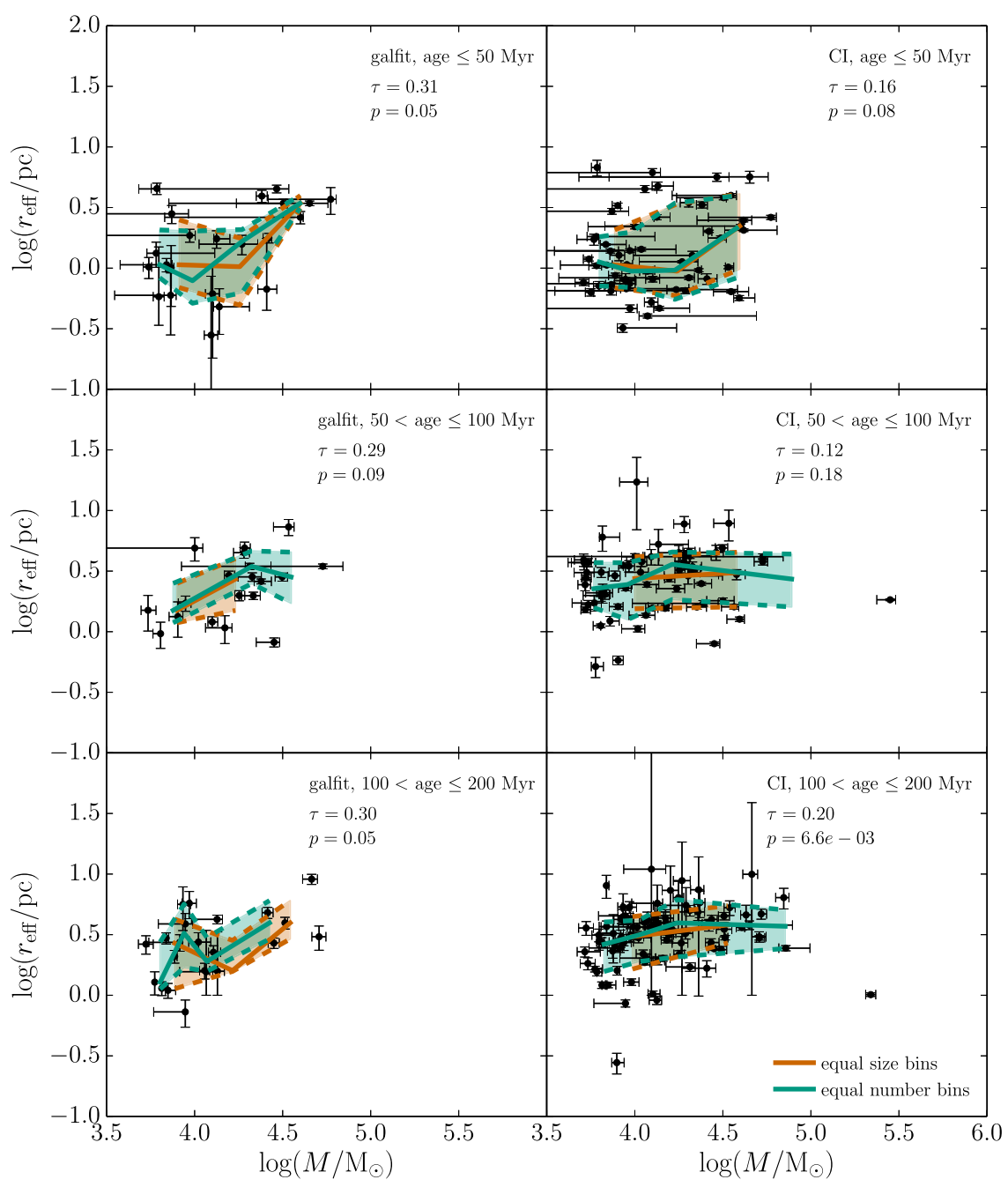

Figure 10. Effective radius as a function of cluster mass for NGC 1313 in three bins in cluster age, 0-50 Myr, 50-100 Myr, and 100-200 Myr. The left column shows the effective radii measured with GALFIT, and the right column shows those estimated from CI values. The solid lines, dashed lines, and shaded regions are the same as in Figure 7. The Kendall $\tau$ correlation statistic and associated $p$ value are given in each panel.

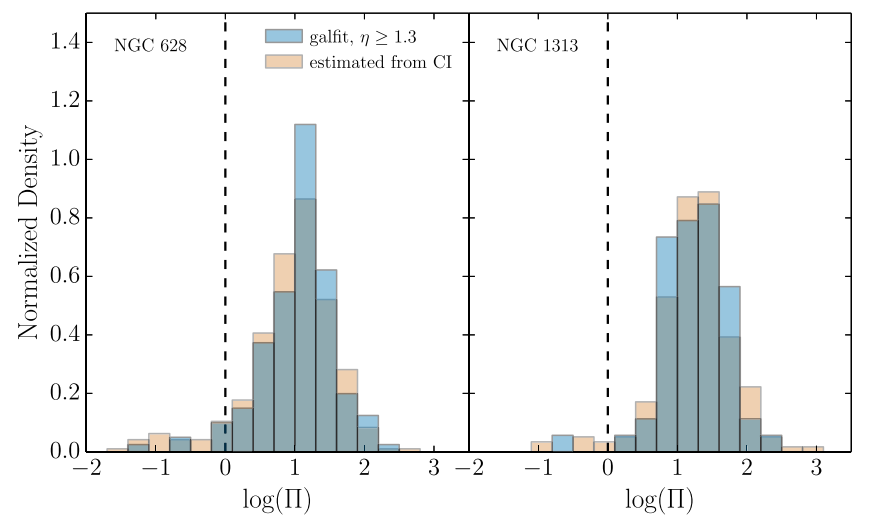

Figure 11. Distribution of dynamical age, $\Pi$, of clusters in NGC 628 (left) and NGC 1313 (right). Dynamical ages calculated using effective radii from GALFIT fits are plotted as blue histograms, and those using CI-estimated effective radii are plotted as orange histograms. The vertical dashed lines separate the bound $(\log (\Pi)>0)$ and unbound $(\log (\Pi)<0)$ objects.

For each cluster located at a galactocentric distance $>2.4 \mathrm{kpc}$ in NGC 628, we estimate the angular speed of the cluster from the rotation velocity of the disk and the galactocentric distance of the cluster, $\omega=v_{\text {rot }} / d_{\mathrm{gc}}$. We find Jacobi radii of 14-61 pc for clusters in the GALFIT sample and $14-88 \mathrm{pc}$ for the CI-estimated sample. In Figure 12, we plot the distribution of half-mass to Jacobi radii ratios for the clusters in NGC 628. The blue histogram shows clusters in the GALFIT sample, and the orange histogram shows those in the CI-estimated sample. We find that the ratio $r_{\mathrm{hm}} / r_{\mathrm{J}}$ takes on values between $\sim 0.02$ and 0.6 ( 0.7 for the CI-estimated sample). About $40 \%$ of the clusters have $r_{\mathrm{hm}} / r_{\mathrm{J}}<0.15$, and $60 \%$ have $r_{\mathrm{hm}} / r_{\mathrm{J}}<0.2$, meaning that about half of the clusters are tidally underfilling while the other half are filling their Roche volumes. We also find a slight, but significant, according to Kendall's $\tau$, positive correlation between cluster age and $r_{\mathrm{hm}} / r_{\mathrm{J}}$, suggesting that the older clusters in NGC 628 are more likely to be tidally filling. These results are very similar to those from M83 in Paper I.

\section{Discussion and Conclusions}

In this work, we have built upon the findings for YMCs in M83 described in Paper I using YMC samples from two galaxies in the LEGUS survey, NGC 628 and NGC 1313. Using similar techniques and data sets, we find strong 


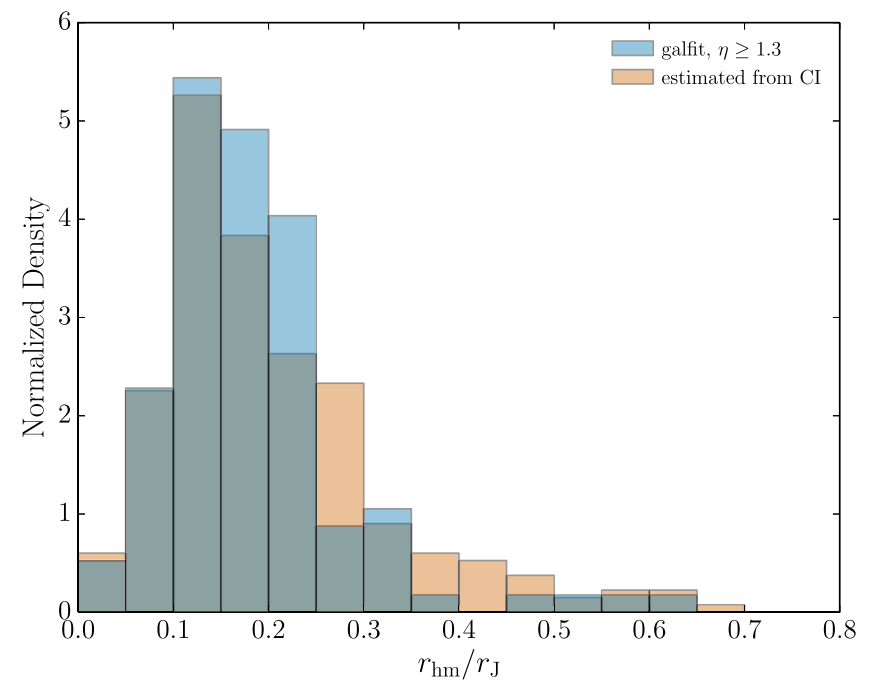

Figure 12. Distribution of the ratio of half-mass radii $\left(r_{\mathrm{hm}}\right)$ to Jacobi radii $\left(r_{\mathrm{J}}\right)$ for clusters in NGC 628. Ratios calculated using effective radii from GALFIT fits are plotted as a blue histogram, and those using CI-estimated effective radii are plotted as an orange histogram.

agreement between the distributions of effective radii and EFF power-law index for YMCs in these three galaxies. We also find very similar results when comparing the structural parameters to other cluster properties, such as age, mass, and galactocentric distance. In addition, we introduce a new technique to measure effective radii of YMCs that utilizes the CI and agrees well with the GALFIT method for fitting radial intensity profiles. Although the YMCs in M83 span a slightly different age and mass range than those in NGC 628 and NGC 1313, the similarity of our results for these three systems suggests that the galaxy environment has little effect on the structural parameters of the YMC populations.

One important result of this work is that the vast majority of YMCs with ages of $\geqslant 10$ Myr for which we are able to measure effective radii are very likely to be gravitationally bound at the present time, given that their ages exceed their dynamical times by a significant margin (Section 4.4). This result shows that the sample selection techniques used by LEGUS, and especially the visual inspection step, which rejects many spurious sources, are successful at producing catalogs of bona fide bound star clusters (visual inspection class 1 and 2). Much of the disagreement in the literature over the properties of cluster populations in nearby galaxies has stemmed from the methods used to define the samples for study. Our results strongly support the notion that careful selection of centrally concentrated, symmetric sources that are extended relative to the stellar PSF is the best method for finding bound, potentially long-lived star clusters.

Because the structures of YMCs derived from this work are similar to those for M83, the astrophysical implications are similar as well. First, it is likely that many of the YMCs in the two LEGUS galaxies are not tidally limited. We find that estimates of the Jacobi radii suggest that about half of the clusters in NGC 628 are underfilling their Roche volumes, while the other half appear to be filling them, and that they appear to be more likely to fill their Roche volumes as they get older. Therefore, the tidal field of NGC 628 may be influencing the structure of some of its YMCs, but overall the effect appears to be minor. In NGC 1313, we are unable to estimate
Jacobi radii, but the other results indicate the tidal field also does not have a significant influence on its clusters.

The effective radii of clusters that are not tidally limited can expand gradually under the influence of stellar mass loss, twobody relaxation, and possibly interactions with GMCs (Heggie \& Hut 2003; Portegies Zwart et al. 2010). Consistent with this expectation, we observe an overall increase in median radius as our clusters age from a few to $200 \mathrm{Myr}$ old, suggesting that many of the star clusters in our samples are still expanding to fill their tidal radii. Similarly, we also would expect to see a stronger correlation between effective radius and galactocentric distance if star clusters were significantly affected by the tidal field of their host galaxy (e.g., Madrid et al. 2012; Puzia et al. 2014).

Recently, the combination of tidal shocks by passing GMCs and two-body relaxation have been invoked to explain the near constancy of effective radii in YMCs by Gieles \& Renaud (2016). Two-body relaxation causes clusters to expand while GMC interactions act to make them contract, in a balancing act that results in a near-constant effective radius as a function of cluster mass, $r_{\mathrm{hm}} \propto M^{1 / 9}$. Including other effects, such as stellar mass loss and binary stars, would be an interesting next step. We find weak relationships between radius and mass for clusters in both of the LEGUS galaxies studied here, in general agreement with the prediction of Gieles \& Renaud (2016). Assuming YMCs are modern-day proto-GCs, the striking similarity between their distributions of effective radii implies that some mechanisms must balance to allow bound star clusters to remain roughly the same size for very long timescales.

These observations are associated with program \# 13364. Support for program \# 13364 was provided by NASA through a grant from the Space Telescope Science Institute. D.A.G. kindly acknowledges financial support by the German Research Foundation (DFG) through grant GO1659/3-2. M.F. acknowledges support by the Science and Technology Facilities Council (grant number ST/L00075X/1).

Facility: HST (ACS/WFC, WFC3/UVIS).

Software: SourceExtractor (Bertin \& Arnouts 1996), Yggdrasil (Zackrisson et al. 2011), Starburst99 (Leitherer et al. 1999), GALFIT (Peng et al. 2002, 2010), IRAF, scipy, baolab (Larsen 1999).

\section{Appendix \\ Kendall's $\tau$}

We use the Kendall's $\tau$ correlation test extensively in this paper, so a more in-depth explanation of its definition and use is warranted. The following draws heavily from Feigelson \& Jogesh Babu (2012) and Ivezić et al. (2014). The purpose of the Kendall's $\tau$ correlation test is to determine if a correlation exists between sets of paired measurements, $\left(X_{1}, Y_{1}\right), \ldots$, $\left(X_{n}, Y_{n}\right)$. This is achieved by comparing the number of concordant pairs, for which $\left(Y_{i}-Y_{j}\right) /\left(X_{i}-X_{j}\right)>0$, with the number of discordant pairs, for which $\left(Y_{i}-Y_{j}\right) /\left(X_{i}-X_{j}\right)<0$. Essentially, to be counted as a concordant pair, the differences in the $X$ and $Y$ values must have the same sign. To be counted as a discordant pair, the differences in the $X$ and $Y$ values must have different signs. If the paired measurements were perfectly correlated (anticorrelated), all of the pairs would be concordant (discordant). If the $X$ values or the $Y$ values are equal for a given pair, this is called a tie. 
We use the kendalltau function within the stats module of the python package scipy to perform the correlation tests. This function defines Kendall's $\tau$ as

$$
\tau=\frac{P-Q}{\sqrt{(P+Q+T)(P+Q+U)}},
$$

where $P$ is the number of concordant pairs, $Q$ is the number of discordant pairs, $T$ is the number of ties only in $X$, and $U$ is the number of ties only in $Y$. If a tie occurs in both the $X$ values and the $Y$ values for the same pair, it is not added to either $T$ or $U$. If all of the pairs are concordant and there are no ties, then $\tau=1$, implying perfect correlation. If all of the pairs are discordant and there are no ties, then $\tau=-1$, implying perfect anticorrelation. As stated by Ivezić et al. (2014), Kendall's $\tau$ can be interpreted as the probability that the two data sets, $X$ and $Y$, are in the same order minus the probability that they are not in the same order.

We choose the Kendall's $\tau$ correlation test because it is nonparametric; that is, it does not require knowledge of the underlying distributions of the data, nor does it assume that any correlation between the two data sets will be linear. Pearson's correlation coefficient, $r$, assumes the data are sampled from a bivariate Gaussian distribution and only looks for linear correlations. Spearman's $\rho$ correlation test is similar in nature to Kendall's $\tau$ and gives very similar results, but it does not approach normality as quickly for small samples as Kendall's $\tau$.

An example of the use of Kendall's $\tau$ correlation test is given in Section 8.8.1 in Feigelson \& Jogesh Babu (2012). They present a data set containing 20 properties of 147 Milky Way GCs and look for a correlation between each pair of properties with Kendall's $\tau$. They find a variety of correlations, both positive and negative, and many are nonlinear. Over half of the correlations are statistically significant, with $|\tau|>0.2$ and $p<0.003$, corresponding to $>3 \sigma$ relationships.

\section{References}

Adamo, A., Ryon, J. E., Messa, M., et al. 2017, ApJ, in press (arXiv:1705. 01588)

Alexander, P. E. R., \& Gieles, M. 2013, MNRAS, 432, L1

Alexander, P. E. R., Gieles, M., Lamers, H. J. G. L. M., \& Baumgardt, H. 2014, MNRAS, 442, 1265

Barmby, P., Kuntz, K. D., Huchra, J. P., \& Brodie, J. P. 2006, AJ, 132, 883

Barmby, P., Perina, S., Bellazzini, M., et al. 2009, AJ, 138, 1667

Bastian, N., Adamo, A., Gieles, M., et al. 2012, MNRAS, 419, 2606

Bastian, N., Cabrera-Ziri, I., Davies, B., \& Larsen, S. S. 2013, MNRAS, 436, 2852

Bastian, N., Gieles, M., Lamers, H. J. G. L. M., Scheepmaker, R. A., \& de Grijs, R. 2005, A\&A, 431, 905

Bertin, E., \& Arnouts, S. 1996, A\&AS, 117, 393

Calzetti, D., Lee, J. C., Sabbi, E., et al. 2015, AJ, 149, 51

Cardelli, J. A., Clayton, G. C., \& Mathis, J. S. 1989, ApJ, 345, 245

Daigle, O., Carignan, C., Amram, P., et al. 2006, MNRAS, 367, 469

de Vaucouleurs, G. 1963, ApJ, 137, 720

Elson, R. A. W., Fall, S. M., \& Freeman, K. C. 1987, ApJ, 323, 54

Fall, S. M., \& Chandar, R. 2012, ApJ, 752, 96
Feigelson, E. D., \& Jogesh Babu, G. 2012, Modern Statistical Methods for Astronomy (Cambridge: Cambridge Univ. Press)

Fouesneau, M., \& Lançon, A. 2010, A\&A, 521, A22

Gieles, M., Heggie, D. C., \& Zhao, H. 2011, MNRAS, 413, 2509

Gieles, M., \& Portegies Zwart, S. F. 2011, MNRAS, 410, L6

Gieles, M., Portegies Zwart, S. F., Baumgardt, H., et al. 2006, MNRAS, 371,793

Gieles, M., \& Renaud, F. 2016, MNRAS, 463, 103

Glatt, K., Grebel, E. K., Gallagher, J. S., III, et al. 2009, AJ, 138, 1403

Harris, W. E. 2009, ApJ, 699, 254

Harris, W. E., Spitler, L. R., Forbes, D. A., \& Bailin, J. 2010, MNRAS, 401, 1965

Heggie, D., \& Hut, P. 2003, The Gravitational Million-Body Problem: A Multidisciplinary Approach to Star Cluster Dynamics (Cambridge: Cambridge Univ. Press)

Hénault-Brunet, V., Gieles, M., Evans, C. J., et al. 2012, A\&A, 545, L1

Hénon, M. 1961, AnAp, 24, 369

Holtzman, J. A., Watson, A. M., Mould, J. R., et al. 1996, AJ, 112, 416

Ivezić, Ž., Connelly, A. J., VanderPlas, J. T., \& Gray, A. 2014, Statistics, Data Mining, and Machine Learning in Astronomy (Princeton, NJ: Princeton Univ. Press)

Jacobs, B. A., Rizzi, L., Tully, R. B., et al. 2009, AJ, 138, 332

Jordán, A., Côté, P., Blakeslee, J. P., et al. 2005, ApJ, 634, 1002

Kissler-Patig, M., Jordán, A., \& Bastian, N. 2006, A\&A, 448, 1031

Larsen, S. S. 1999, A\&AS, 139, 393

Larsen, S. S. 2004, A\&A, 416, 537

Leitherer, C., Schaerer, D., Goldader, J. D., et al. 1999, ApJS, 123, 3

Mackey, A. D., \& Gilmore, G. F. 2003, MNRAS, 338, 85

Madrid, J. P., Hurley, J. R., \& Sippel, A. C. 2012, ApJ, 756, 167

Massey, P., Olsen, K. A. G., Hodge, P. W., et al. 2006, AJ, 131, 2478

Masters, K. L., Jordán, A., Côté, P., et al. 2010, ApJ, 715, 1419

Matthews, L. D., Gallagher, J. S., III, Krist, J. E., et al. 1999, AJ, 118, 208

McLaughlin, D. E., \& van der Marel, R. P. 2005, ApJS, 161, 304

Mora, M. D., Larsen, S. S., Kissler-Patig, M., Brodie, J. P., \& Richtler, T. 2009, A\&A, 501, 949

Olivares, E. F., Hamuy, M., Pignata, G., et al. 2010, ApJ, 715, 833

Pellerin, A., Meyer, M., Harris, J., \& Calzetti, D. 2007, ApJL, 658, L87

Peng, C. Y., Ho, L. C., Impey, C. D., \& Rix, H.-W. 2002, AJ, 124, 266

Peng, C. Y., Ho, L. C., Impey, C. D., \& Rix, H.-W. 2010, AJ, 139, 2097

Peters, W. L., Freeman, K. C., Forster, J. R., Manchester, R. N., \& Ables, J. G. 1994, MNRAS, 269, 1025

Popescu, B., \& Hanson, M. M. 2010, ApJ, 724, 296

Portegies Zwart, S. F., McMillan, S. L. W., \& Gieles, M. 2010, ARA\&A, 48, 431

Puzia, T. H., Paolillo, M., Goudfrooij, P., et al. 2014, ApJ, 786, 78

Ryder, S. D., Staveley-Smith, L., Malin, D., \& Walsh, W. 1995, AJ, 109, 1592

Ryon, J. E., Bastian, N., Adamo, A., et al. 2015, MNRAS, 452, 525

Scheepmaker, R. A., Haas, M. R., Gieles, M., et al. 2007, A\&A, 469, 925

Silva-Villa, E., \& Larsen, S. S. 2011, A\&A, 529, A25

Silva-Villa, E., \& Larsen, S. S. 2012, MNRAS, 423, 213

Smith, L. J., \& Gallagher, J. S. 2001, MNRAS, 326, 1027

Sun, W., de Grijs, R., Fan, Z., \& Cameron, E. 2016, ApJ, 816, 9

Thilker, D. A., Bianchi, L., Meurer, G., et al. 2007, ApJS, 173, 538

Trenti, M., Vesperini, E., \& Pasquato, M. 2010, ApJ, 708, 1598

van den Bergh, S., Morbey, C., \& Pazder, J. 1991, ApJ, 375, 594

Vázquez, G. A., \& Leitherer, C. 2005, ApJ, 621, 695

Webb, J. J., Sills, A., Harris, W. E., et al. 2016, MNRAS, 460, 2129

Whitmore, B. C., Chandar, R., Schweizer, F., et al. 2010, AJ, 140, 75

Whitmore, B. C., Zhang, Q., Leitherer, C., et al. 1999, AJ, 118, 1551

Zackrisson, E., Rydberg, C.-E., Schaerer, D., Östlin, G., \& Tuli, M. 2011, ApJ, 740, 13

Zepf, S. E., Ashman, K. M., English, J., Freeman, K. C., \& Sharples, R. M. 1999, AJ, 118, 752 